\title{
INÉGALITÉ D'OBSERVABILITÉ DU TYPE LOGARITHMIQUE ET ESTIMATION DE LA FONCTION DE COÛT DES SOLUTIONS DES ÉQUATIONS HYPERBOLIQUES
}

\author{
LEILA OUKSEL ${ }^{1}$
}

\begin{abstract}
Résumé. Dans ce travail, nous donnons une estimation logarithmique des données de la solution $u$, d'un problème hyperbolique avec condition aux limites de type Neumann, par la trace de $u$ restreinte à un ouvert du bord, pendant un temps suffisamment grand qui nous permet d'estimer la fonction de coût de ce problème.
\end{abstract}

\begin{abstract}
This work proposes a logarithmic estimation of the initial values of the solution $u$ of a hyperbolic problem, with Neumann boundary conditions, using the trace of $u$ restricted to the neighbourhood of the boundary, during a time sufficiently large for estimating the cost function of the problem.
\end{abstract}

Classification Mathématique. 35B37, 93B05, 93B07, 35L20.

Received November 6, 2005. Revised June 16, 2006.

Published online October 13, 2007.

\section{INTRODUCTION}

Nous nous intéressons ici au problème de contrôle des solutions des équations hyperpoliques. Le cadre fonctionnel a été dégagé par Lions [20] qui a introduit la méthode H.U.M. (Hilbert Uniqueness Method) qui consiste à étudier l'ensemble des conditions initiales d'un problème d'évolution, qui peuvent être annulées en un temps fini $T$ en exerçant un contrôle d'énergie finie sur le bord. Ce résultat repose d'une façon fondamentale sur un théorème d'unicité qui permet de construire un espace de Hilbert dense dans l'espace d'énergie. Ce théorème d'unicité est une conséquence du théorème de Holmgren dans le cadre analytique. Dans le cas d'un opérateur hyperpolique scalaire d'ordre deux, $D_{t}^{2}-A\left(t, x, D_{x}\right)$, Alinhac [1] et Alinhac et Baouendi [2] ont montré que la régularité $C^{\infty}$ des coefficients de l'opérateur elliptique $A$ ne suffit pas pour avoir l'unicité par rapport à une surface de type temps. En effet, il existe deux fonctions de classe $C^{\infty} a(t, x)$ et $u(t, x)$ telles que $\left(D_{t}^{2}-\Delta+a(t, x)\right) u(t, x)=0$ dans un voisinage $V$ de $(t, x)=(0,0)$ et supp $u \subset\left\{x=\left(x_{1}, x^{\prime}\right) ; x_{1} \geq-\delta\left|x^{\prime}\right|\right\}$ où $\delta>0$. Par la suite, il a été observé par Robbiano [28] que lorsque l'opérateur $A$ ne dépend pas du temps, nous avons un résultat d'unicité adapté à la théorie de contrôle. Ce résultat a été amélioré, dans un premier temps, par Hörmander [11] qui a donné une estimation précise et quasiment optimale du temps minimal pendant lequel le contrôle doit agir, puis par Tataru [32,33].

\footnotetext{
Mots Clés. Problème hyberbolique, contrôle, fonction de coût, inégalité de Carleman.

1 Université de Paris Sud, Mathématiques, Bât. 425, 91405 Orsay cedex, France; Leila.Ouksel@math.u-psud.fr, louksel@yahoo.fr
} 
Récemment, Robbiano et Zuily [30] d'une part, et Hörmander [12] et Tataru [34] d'autre part, ont démontré un théorème d'unicité locale pour les opérateurs différentiels, scalaires, à coefficients $C^{\infty}$, partiellement holomorphes, où les hypothèses usuelles de Hörmander (principalement normalité et pseudo-convexité) sont faites uniquement sur le conormal des variables analytiques.

Bardos, Lebeau et Rauch [4] ont montré qu'il existe un lien entre les rayons de la géométrie optique et le problème de la contrôlabilité exacte, une condition de contrôle géométrique, suffisante et presque nécessaire, pour obtenir l'observabilité de ces problèmes. Sans cette condition de contrôle géométrique, Robbiano [29] prouve un estimateur d'observabilité logarithmique (mais avec T suffisamment large à cause de la vitesse de propagation finie) et démontre comment l'utiliser afin d'obtenir un résultat de contrôle approximatif avec une estimation du coût du contrôle.

Les problèmes de contrôlabilité et d'observabilité ont été également étudiés dans le cas des problèmes paraboliques; Fursikov, Imanivilov [10] et Lebeau et Robbiano [18] ont montré une contrôlabilité exactement nulle, sans restriction sur le temps de contrôle ou sur le support de la fonction de contrôle se sont inspirés de la méthode de Russell [31], qui a introduit une transformation pour montrer que l'équation de chaleur entraine une contrôlabilité nulle pour toutes les valeurs du temps, et ce à partir de la contrôlabilité exacte de l'équation d'onde pour un certain intervalle (certaines valeurs) du temps. Fernandez, Cara et Zuazua [9] ont également démontré un estimateur d'observabilité, explicite dans le temps. Récemment, Miller [23,24] a montré la contrôlabilité nulle pour un temps assez petit et dans des domaines non bornés. Ces problèmes de contrôlabilité et d'observabilité ont encore été étudiés par Lebeau [16], Fabre [8], Lasiecka et Tariggiani [15] et Burq [5], ainsi que Machtyngier [22] et Phung [25] pour l'équation de Schrödinger, dans le cas où le contrôle agit sur une partie du bord.

Dans ce travail, nous reprenons les estimations de Robbiano [28] pour donner un résultat quantitatif d'unicité. Plus précisement, soit $u$ la solution d'un problème hyperbolique dans un ouvert avec la dérivée normale nulle au bord, et si $u$ est nulle sur un ouvert du bord pendant un temps suffisamment grand, alors $u$ est nulle et nous prouvons une majoration des données de $u$ par la trace de $u$ en norme $L^{2}$ restreinte à un ouvert du bord pendant un temps suffisamment grand. Une estimation du même type que celle obtenue ici avec la norme de la trace de $u$ sur le bord dans $H^{1}$ résulterait des travaux précédents sur le sujet. L'estimation naturelle pour le problème de Neumann et avec la norme $L^{2}$ de la trace de $u$ sur le bord et ceci nous a conduit à prouver une estimation de Carleman adaptée (voir Prop. 2.2.1). Cette majoration n'est pas linéaire comme dans le cas traité par Bardos, Lebeau et Rauch [4], mais logarithmique. Ce type d'estimation a été donné par John [14] dans le cadre analytique. Dans les cas où l'unicité se démontre par une inégalité de Carleman, Isakov [13] et Bahouri [3] ont montré que nous obtenons ce que John appelle des inégalités de type Hölder. En reprenant les mêmes idées, nous prouvons que les estimations de Robbiano [28] donnent des estimations logarithmiques des données par la trace de $u$, puis nous donnons une estimation de la fonction de coût notée $C(\epsilon)$ de ce problème du type $C \exp \left(C / \epsilon^{2}\right)$.

Ce type d'estimations logarithmiques a également été utilisé pour des problèmes paraboliques, Phung [26] a donné la contrôlabilité approchée de l'equation de la chaleur avec un potentiel, et pour l'équation de Schrödinger, Phung [25] a montré une estimation logarithmique en absence de contrôle géometrique. Duychaerts [6], Zhang et Zuazua [37] et Duyckaerts, Zhang et Zuazua [7] utilisent ces estimations pour l'interaction des problèmes d'ondes-chaleurs qui donne le taux de décroissance logarithmique lorsque l'équation de la chaleur ne satisfait pas la condition de contrôle géometrique.

\section{Remarques}

- Probablement, il est possible d'avoir les mêmes résultats de ce travail quand nous abaissons la régularité des coefficients du problème hyperbolique, cela nécessiterait de remplacer l'utilisation du calcul pseudodifférentiel par d'autres outils, adaptés à des opérateurs à régularités limitées.

- Les résultats sur le contrôle pour l'équation des ondes ont été obtenus dans le cadre de la contrôlabilité exacte, soit par des méthodes de multiplicateur, soit par des techniques microlocales (propagation d'onde ou mesures microlocales) et sont équivalents à des estimations linéaires d'observabilité. 
Lebeau [17] a prouvé dans un cadre analytique des résultats très précis sur l'espace atteignable et ont pour conséquence des estimations logarithmiques d'observabilité. Dans le cadre $C^{\infty}$, des techniques issues des inégalites de Carleman, des estimations d'observabilité du même type peuvent être prouvées, mais avec des puissances moins bonnes. La transition entre les deux phénomènes, qu'on pourrait un peu rapidement résumer par les hautes fréquences pour la contrôlabilité exacte et par moyennes fréquences dans le deuxième cas, n'est pas comprise.

Pour des systèmes couplés, des estimations d'observabilité polynomiales ont été prouvées, mais ce type de phénomène semble plutôt lié au couplage qu'à la transition hautes-moyennes fréquences (voir les travaux de Rauch, Zhang et Zuazua [27], Zhang et Zuazua [35,36]).

\section{HypothÈSES ET RÉSULTATS}

Soit $\Omega$ un ouvert borné et connexe de $\mathbb{R}^{n}$, de frontière $\partial \Omega$ de classe $C^{\infty}$, soit $\Gamma$ une partie ouverte non vide de $\partial \Omega$, on note :

$$
\Omega_{T}=(0, T) \times \Omega \quad \text { et } \quad \Gamma_{T}=(0, T) \times \Gamma, \quad T>0 .
$$

Soit $A\left(x, D_{x}\right)$ un opérateur elliptique de la forme suivante :

$$
A\left(x, D_{x}\right)=\sum_{1 \leq j, k \leq n} D_{j}\left(a_{j, k}(x) D_{k}\right)+\sum_{j=1}^{n} D_{j} a_{j}(x)+a_{0}(x) \quad \text { où } \quad D_{j}=\frac{1}{i} \frac{\partial}{\partial x_{j}} .
$$

On suppose que $\left(a_{j, k}(x)\right)_{1 \leq j, k \leq n}$ est une matrice symétrique définie positive et qu'il existe une constante positive $C_{1}$ telle que, pour tout $x \in \Omega$ et tout $\xi \in \mathbb{R}^{n}$, on ait :

$$
\sum_{1 \leq j, k \leq n} a_{j, k}(x) \xi_{j} \xi_{k} \geq C_{1}|\xi|^{2}
$$

les fonctions $a_{j, k}, a_{j}$ et $a_{0}$ vérifiant :

$$
\begin{gathered}
a_{j, k} \in C^{\infty}(\bar{\Omega}) \quad \text { pour } \quad 1 \leq j, k \leq n \\
a_{j} \in L^{\infty}(\bar{\Omega}) \quad \text { pour } \quad j=0, \ldots, n,
\end{gathered}
$$

il existe donc une constante positive $C_{2}$ telle que, pour tout $x \in \Omega$ et tout $\xi \in \mathbb{R}^{n}$, on ait :

$$
C_{2}|\xi|^{2} \geq \sum_{1 \leq j, k \leq n} a_{j, k}(x) \xi_{j} \xi_{k} .
$$

On note $B=\partial_{\eta_{A}}=-\sum_{1 \leq j, k \leq n} a_{j, k}(x) \eta_{j} D_{k}$ la dérivée conormale extérieure à $\Omega$ où $\eta(x)=\left(\eta_{j}(x)\right)_{1 \leq j \leq n}$ est la normale unitaire extérieure à $\Omega$ en $x$ et $C=\partial_{\eta_{A}}-\sum_{1 \leq j \leq n} a_{j}(x) \eta_{j}$.

- On s'interesse ici au problème hyperbolique d'évolution avec contrôle du type Neumann sur la frontière du problème.

On note

$$
W_{0}=H^{1}(\Omega) \oplus L^{2}(\Omega) ; \quad W_{-1}=L^{2}(\Omega) \oplus\left(H^{1}\right)^{\prime}(\Omega)
$$

où $\left(H^{1}\right)^{\prime}(\Omega)$ est le dual de $H^{1}(\Omega)$. Les espaces $W_{0}$ et $W_{-1}$ sont en dualité par la forme bilinéaire suivante :

$\mathrm{Si} \vec{v}=\left(v_{0}, v_{1}\right) \in W_{-1}, \vec{u}=\left(u_{0}, u_{1}\right) \in W_{0}$ alors $(\vec{v}, \vec{u})=\left(u_{0} \mid v_{1}\right)-\left(u_{1} \mid v_{0}\right)$ où (.|.) est soit le produit scalaire dans $L^{2}(\Omega)$, soit la dualité entre $H^{1}(\Omega)$ et $\left(H^{1}\right)^{\prime}(\Omega)$. 
Pour $\vec{u}=\left(u_{0}, u_{1}\right) \in W_{0}$ on note encore $u(t, x)$ la solution appartenant à $C^{0}\left(\mathbb{R}, H^{1}(\Omega)\right) \cap C^{1}\left(\mathbb{R}, L^{2}(\Omega)\right)^{1}$ du problème homogène direct :

$$
\left\{\begin{array}{lll}
{\left[D_{t}^{2}-A(x, D)\right] u=0} & \text { dans } & \mathbb{R} \times \Omega \\
u_{\mid t=0}=u_{0} & \text { dans } & \Omega \\
\partial_{t} u_{\mid t=0}=u_{1} & \text { dans } & \Omega \\
C u=0 & \text { sur } & \mathbb{R} \times \partial \Omega
\end{array}\right.
$$

Par ailleurs, si $C \phi=0$ sur $\partial \Omega$ et $\left.\operatorname{supp} \phi\right|_{\partial \Omega} \subset \Gamma$ (pour $\phi$ et $\psi$ suffisamment régulières), on a :

$$
\int_{\Omega}\left[A \phi \bar{\psi}-\phi \overline{A^{*} \psi}\right] \mathrm{d} x=\int_{\Gamma} \phi \partial_{\eta_{A}} \bar{\psi} \mathrm{d} \sigma .
$$

La formule (1.0.2) permet de prouver par la méthode de transposition ( $c f$. [21]) que le problème rétrograde non homogène suivant est bien posé.

Soit $g \in L^{2}\left(\Gamma_{T}\right)$, on peut alors résoudre :

$$
\left\{\begin{array}{lll}
{\left[D_{t}^{2}-A^{*}(x, D)\right] v=0} & \text { dans } & \mathbb{R} \times \Omega \\
\left.v\right|_{t=T}=\left.\partial_{t} v\right|_{t=T}=0 & \text { dans } & \Omega \\
B v=g \mathbf{1}_{\Gamma_{T}} & \text { sur } & \mathbb{R} \times \partial \Omega
\end{array}\right.
$$

avec $v \in C^{0}\left(\mathbb{R}, L^{2}(\Omega)\right) \cap C^{1}\left(\mathbb{R},\left(H^{1}(\Omega)\right)^{c}\right)$.

On définit ainsi $\mathcal{S}$ par :

$$
\begin{aligned}
\mathcal{S}: L^{2}\left(\Gamma_{T}\right) & \longrightarrow W_{-1} \\
g & \mapsto \mathcal{S} g=\vec{v}=\left(v_{\mid t=0}, \partial_{t} v_{\mid t=0}\right) .
\end{aligned}
$$

On pose $F=\operatorname{Im}(\mathcal{S})$ (tel que $\|\vec{v}\|_{F}=\inf \left\{\|g\|_{L^{2}\left(\Gamma_{T}\right)} ; \mathcal{S} g=\vec{v}\right\}$ ), ceci nous permet de dire que si $\vec{v} \in F$, il existe $g \in L^{2}\left(\Gamma_{T}\right)$ tel que $\vec{v}=\mathcal{S}(g)$, solution du problème d'évolution :

$$
\left\{\begin{array}{lll}
{\left[D_{t}^{2}-A(x, D)\right] v=0} & \text { dans } & \mathbb{R} \times \Omega \\
v(0, x)=v_{0}, \partial_{t} v(0, x)=v_{1} & \text { dans } & \Omega \\
B v=g \mathbf{1}_{\Gamma_{T}} & \text { sur } & \mathbb{R} \times \partial \Omega
\end{array}\right.
$$

qui est contrôlable par définition.

Les résultats principaux de ce papier sont :

Théorème 1.0.1. Il existe des constantes $T>0$ et $C>0$ telles que pour tout $\vec{u} \in W_{0}$ on ait

$$
\|\vec{u}\|_{W_{-1}} \leq \frac{C\|\vec{u}\|_{W_{0}}}{\left[\log \left(2+\frac{\|\vec{u}\|_{W_{0}}}{\left\|\left.u\right|_{\Gamma_{T}}\right\|_{L^{2}\left(\Gamma_{T}\right)}}\right)\right]^{\frac{1}{2}}} .
$$

Théorème 1.0.2. Il existe une constante $C>0$ telle que pour tout $\vec{u} \in W_{0},\|\vec{u}\|_{W_{0}} \leq 1$, pour tout $\epsilon>0$, il existe $\vec{y} \in F$ et $\vec{z} \in W_{-1}$ tels que $\vec{u}=\vec{z}+\vec{y}$ avec $\|\vec{y}\|_{F} \leq C \mathrm{e}^{\frac{C}{\epsilon^{2}}},\|\vec{z}\|_{W_{-1}} \leq \epsilon$.

\footnotetext{
${ }^{1}$ Voir [21], page 286.
} 


\section{Remarque 1.0.3.}

$1-F$ dépend de $T$. Pour le Théorème 1.0.2, il s'agit du $F$ défini avec le temps $T$ du Théorème 1.0.1.

2 - On peut réécrire le Théorème 1.0.2 en utilisant les fonctions de coût.

On introduit la fonction de coût $C(\epsilon)$ de la manière suivante :

$$
\text { Posons } \quad C_{\vec{u}}(\epsilon)=\inf \left\{\|\vec{y}\|_{F} ; \vec{u}=\vec{y}+\vec{z},\|\vec{z}\|_{W_{-1}} \leq \epsilon\right\},
$$

la fonction de coût est alors définie par :

$$
C(\epsilon)=\sup \left\{C_{\vec{u}}(\epsilon) ; \vec{u} \in W_{0},\|\vec{u}\|_{W_{0}} \leq 1\right\} .
$$

Corollaire 1.0.4. Il existe une constante $C>0$, telle que

$$
C(\epsilon) \leq C \mathrm{e}^{\frac{C}{\epsilon^{2}}} \text { pour tout } \epsilon>0 .
$$

- On va commencer par prouver le Théorème 1.0.2 en supposant démontrer le Théorème 1.0.1.

Preuve du Théorème 1.0.2. D'abord on définit l'opérateur continu suivant:

$$
\begin{aligned}
K: W_{0} & \rightarrow L^{2}\left(\Gamma_{T}\right) \\
\vec{u} & \mapsto K \vec{u}=u \mathbf{1}_{\Gamma_{T}},
\end{aligned}
$$

puis on rappelle le lien entre les deux opérateurs $\mathcal{S}$ et $K$; pour cela on applique la formule (1.0.2) à $\Phi=u$, solution du problème (1.0.1) et $\Psi=v$ solution du problème (1.0.3). On remplace $A u$ par $D_{t}^{2} u$ et $A^{*} v$ par $D_{t}^{2} v$, puis on intégre entre 0 et $T$, en effectuant une intégration par parties :

$$
\langle\mathcal{S}(g), \vec{u}\rangle=\int_{0}^{T} \int_{\Gamma} K(\vec{u})(x, t) \overline{g(x, t)} \mathrm{d} \sigma \mathrm{d} t .
$$

On note $\Lambda: W_{-1} \longrightarrow W_{0}$ l'opérateur tel que pour tout $\vec{u} \in W_{0}$ et $\vec{v} \in W_{-1},\langle\vec{u}, \vec{v}\rangle=(\Lambda \vec{v}, \vec{u})_{W_{0}}$ où $(,)_{W_{0}}$ est le produit scalaire de $W_{0}$.

L'opérateur $\Lambda$ est bien défini (d'après le théorème de représentation de Riesz).

On note $\mathcal{B}: W_{0} \rightarrow W_{0} ; \mathcal{B}=\Lambda \circ \mathcal{S} \circ K$. En posant $g=K \vec{u}$ dans (1.0.5) on trouve :

$$
(\mathcal{B} \vec{u}, \vec{u})_{W_{0}}=\int_{0}^{T} \int_{\Gamma}|K(\vec{u})|^{2} \mathrm{~d} \sigma \mathrm{d} t .
$$

Ce qui implique que l'opérateur $\mathcal{B}$ est borné, positif et autoadjoint.

On note $E_{\lambda}$ le projecteur spectral associé à $\mathcal{B}$, on a supp $\mathrm{d} E_{\lambda} \subset[0,\|K\|]$ et :

$$
\vec{u}=\int_{0}^{+\infty} \mathrm{d} E_{\lambda} \vec{u} \quad \text { et } \quad \mathcal{B} \vec{u}=\int_{0}^{+\infty} \lambda \mathrm{d} E_{\lambda} \vec{u} .
$$

On note $C_{n}=C \mathrm{e}^{-^{\mathrm{e}^{n}}}$ avec $C$ assez grand qui sera choisi ultérieurement et

$$
G_{n} \vec{u}=\int 1_{\left(C_{n+1}, C_{n}\right)} \mathrm{d} E_{\lambda} \vec{u}=E_{C_{n}}(\vec{u})-E_{C_{n+1}}(\vec{u})
$$


donc $\vec{u}=\sum_{n \geq 0} G_{n} \vec{u}$ si $C \mathrm{e}^{-1} \geq\|K\|$; de plus on a :

$$
\begin{aligned}
\left\|K G_{n} \vec{u}\right\|_{L^{2}\left(\Gamma_{T}\right)}^{2} & =\left(\mathcal{B} G_{n} \vec{u}, G_{n} \vec{u}\right)_{W_{0}} \\
& =\int \lambda 1_{\left(C_{n+1}, C_{n}\right]}\left(\mathrm{d} E_{\lambda} G_{n} \vec{u}, G_{n} \vec{u}\right)_{W_{0}},
\end{aligned}
$$

on a clairement $C_{n+1}\left\|G_{n} \vec{u}\right\|_{W_{0}}^{2} \leq\left\|K G_{n} \vec{u}\right\|_{L^{2}\left(\Gamma_{T}\right)}^{2} \leq C_{n}\left\|G_{n} \vec{u}\right\|_{W_{0}}^{2}$.

Notons $d_{n}=\frac{1}{C} \mathrm{e}^{\mathrm{e}^{n}}=C_{n}^{-1}$, on obtient

$$
d_{n}\left\|K G_{n} \vec{u}\right\|_{L^{2}\left(\Gamma_{T}\right)}^{2} \leq\left\|G_{n} \vec{u}\right\|_{W_{0}}^{2} \leq d_{n+1}\left\|K G_{n} \vec{u}\right\|_{L^{2}\left(\Gamma_{T}\right)}^{2} .
$$

On note l'espace $F^{\prime}$, dual de $F$ qui est le complété de $W_{0}$ pour la norme $\|K \vec{u}\|_{L^{2}\left(\Gamma_{T}\right)}$.

- Estimation de la norme de $G_{n} \vec{u}$ dans $W_{-1}$.

Il découle du Théorème 1.0.1 :

$$
\left\|G_{n} \vec{u}\right\|_{W_{-1}} \leq \frac{C\left\|G_{n} \vec{u}\right\|_{W_{0}}}{\left[\log \left(2+\frac{\left\|G_{n} \vec{u}\right\|_{W_{0}}}{\left\|K G_{n} \vec{u}\right\|_{L^{2}\left(\Gamma_{T}\right)}}\right)\right]^{\frac{1}{2}}}
$$

de l'inégalité (1.0.7) on déduit,

$$
\left\|G_{n} \vec{u}\right\|_{W_{-1}} \leq C \mathrm{e}^{-\frac{n}{2}}\left\|G_{n} \vec{u}\right\|_{W_{0}} .
$$

On munit $W_{0}$ de la norme : $\sum_{n \geq 0} \mathrm{e}^{-\frac{n}{2}}\left\|G_{n} \vec{u}\right\|_{W_{0}}$ et on pose $H$ son complété pour cette norme (on note $\|\cdot\|_{H}$ la norme de cet espace).

On a, grâce à (1.0.8), la propriété : $H \subset W_{-1}$.

On munit également $W_{0}$ de la norme : $\sum_{n \geq 0} \mathrm{e}^{-\frac{\mathrm{e}^{n+1}}{2}}\left\|G_{n} \vec{u}\right\|_{W_{0}}$ et on pose $I$ son complété pour cette norme (on note $\|\cdot\|_{I}$ la norme de cet espace).

Grâce à (1.0.7), on a $F^{\prime} \subset I$, on en déduit alors le diagramme suivant :

$$
\begin{gathered}
W_{0} \subset H \subset F^{\prime} \subset I \\
\cap \\
W_{-1} .
\end{gathered}
$$

Par la dualité entre $W_{0}$ et $W_{-1}$, on obtient

$$
\begin{gathered}
I^{\prime} \subset F \subset H^{\prime} \subset W_{-1} \\
\cup \\
W_{0} .
\end{gathered}
$$

- La description de $H^{\prime}$ et de $I^{\prime}$ est obtenue comme suit :

On note $\widetilde{E_{\lambda}}$ l'opérateur dans $W_{-1}$ déduit par dualité de $E_{\lambda}$, c'est-à-dire pour tout $\vec{v} \in W_{-1}$ et $\vec{u} \in W_{0}$ on a

$$
\left\langle\vec{v}, E_{\lambda} \vec{u}\right\rangle=\left\langle\widetilde{E_{\lambda}} \vec{v}, \vec{u}\right\rangle
$$

et notant

$$
\widetilde{G_{n}}=\int 1_{\left(C_{n+1}, C_{n}\right)} \mathrm{d} \widetilde{E_{\lambda}}
$$


on a clairement, si $k \neq n,\left\langle\widetilde{G}_{n} \vec{v}, G_{k} \vec{u}\right\rangle=0$.

Il est alors facile de vérifier que

$$
H^{\prime}=\left\{\vec{v} \in W_{-1} ; \sup _{n \geq 0} \mathrm{e}^{\frac{n}{2}}\left\|\widetilde{G_{n}} \vec{v}\right\|_{W_{-1}}<+\infty\right\}
$$

et

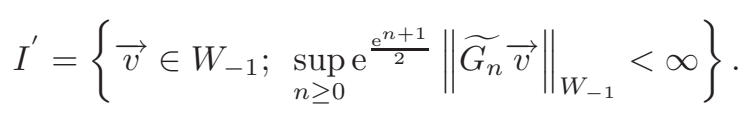

Soit $\vec{u} \in W_{0},\|\vec{u}\|_{W_{0}} \leq 1$, puisque $W_{0} \subset H^{\prime}$ alors

$$
\left\|\widetilde{G_{n}} \vec{u}\right\|_{W_{-1}} \leq C \mathrm{e}^{-\frac{n}{2}}\|\vec{u}\|_{W_{0}} \leq C \mathrm{e}^{-\frac{n}{2}}
$$

d'autre part on a $\vec{u}=\sum_{n \geq 0} \widetilde{G_{n}} \vec{u}$.

On note $\vec{y}=\sum_{n=0}^{N} \widetilde{G_{n}} \vec{u}$ et $\vec{z}=\sum_{n=N+1}^{+\infty} \widetilde{G_{n}} \vec{u}$, où $N$ sera choisi plus loin.

En vertu de (1.0.10), on obtient (où les constantes $C$ peuvent changer de ligne en ligne) :

$$
\sup _{n \geq 0} \mathrm{e}^{\frac{\mathrm{e}^{n+1}}{2}}\left\|\widetilde{G_{n}} \vec{y}\right\|_{W_{-1}} \leq \sup _{n \geq 0} \sum_{n=0}^{N} C \mathrm{e}^{\frac{\mathrm{e}^{n+1}}{2}} \cdot \mathrm{e}^{-\frac{\mathrm{e}^{n}}{2}} \leq C \mathrm{e}^{C \mathrm{e}^{N}}
$$

et $\|\vec{z}\|_{W_{-1}} \leq \sum_{n=N+1}^{+\infty}\left\|\widetilde{G_{n}} \vec{u}\right\|_{W_{-1}} \leq \sum_{n=N+1}^{+\infty} C \mathrm{e}^{-\frac{n}{2}} \leq C \mathrm{e}^{-\frac{N+1}{2} 2}$.

Pour $\epsilon$ donné, soit $N$ tel que $C \mathrm{e}^{-\frac{N}{2}}>\epsilon \geq C \mathrm{e}^{-\frac{(N+1)}{2}}$, il en résulte alors $\|\vec{z}\|_{W_{-1}} \leq \epsilon$ et $\|\vec{y}\|_{I^{\prime}} \leq C \mathrm{e}^{\frac{C}{\epsilon^{2}}}$, ce qui prouve le Théorème 1.0.2 puisque $I^{\prime} \subset F$.

\section{InÉGAlitÉS D'Interpolation ET DE CARLEMAN}

\subsection{Inégalité d'interpolation}

Soient $\left.s_{0}>0, X=\right]-s_{0}, s_{0}[\times \Omega, \partial X=]-s_{0}, s_{0}\left[\times \partial \Omega\right.$ et $v \in C^{\infty}(X)$ la solution de

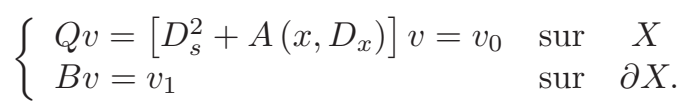

Soit $\Gamma$ un ouvert non vide de $\partial \Omega$, on note $\left.\Gamma_{s_{0}}=\right]-s_{0}, s_{0}[\times \Gamma$ et $Y=]-\frac{s_{0}}{2}, \frac{s_{0}}{2}[\times \Omega$.

Théorème 2.1.1. Il existe $C>0$ et $\mu \in] 0,1[$ tels que pour toute solution $v$ de (2.1.1), on a

$$
\|v\|_{H^{1}(Y)} \leq C\left(\|Q v\|_{L^{2}(X)}+\|B v\|_{L^{2}(\partial X)}+\left\|\left.v\right|_{\Gamma_{S_{0}}}\right\|_{L^{2}(\partial X)}\right)^{\mu}\left(\|v\|_{H^{1}(X)}\right)^{1-\mu}
$$

Remarque 2.1.2. Une inégalité de ce type a déjà été prouvée dans [18] pour une autre condition au bord. La preuve du Théorème 2.1.1 repose sur les résultats de [18,19].

\footnotetext{
${ }^{2}$ Car $=\sum_{n \geq 0} \mathrm{e}^{-\frac{n}{2}}$ est une série géométrique de raison $\mathrm{e}^{-\frac{1}{2}}$.
} 
Proposition 2.1.3. Il existe $C>0, \mu \in] 0,1\left[\right.$, un ouvert $U$ non vide de $\Omega$ et $\epsilon>0$ tels que pour tout $v \in H^{2}(X)$, on ait, en posant $\left.U_{0}=\right]-\epsilon, \epsilon[\times U$, les inégalités d'interpolations

$$
\begin{gathered}
\|v\|_{L^{2}\left(U_{0}\right)} \leq C\left(\|Q v\|_{L^{2}(X)}+\|B v\|_{L^{2}(\partial X)}+\left\|\left.v\right|_{\Gamma_{S_{0}}}\right\|_{L^{2}(\partial X)}\right)^{\mu}\left(\|v\|_{H^{1}(X)}\right)^{1-\mu} \\
\|v\|_{H^{1}(Y)} \leq C\left(\|Q v\|_{L^{2}(X)}+\|B v\|_{L^{2}(\partial X)}+\|v\|_{L^{2}\left(U_{0}\right)}\right)^{\mu}\left(\|v\|_{H^{1}(X)}\right)^{1-\mu}
\end{gathered}
$$

Preuve du Théorème 2.1.1.

- Si $\|v\|_{L^{2}\left(U_{0}\right)} \leq\|Q v\|_{L^{2}(X)}+\|B v\|_{L^{2}(\partial X)}$, alors (2.1.4) nous donne (2.1.2).

- Sinon (2.1.4) s'écrit $\|v\|_{H^{1}(Y)} \leq C^{\prime}\|v\|_{L^{2}\left(U_{0}\right)}^{\mu}\|v\|_{H^{1}(X)}^{1-\mu}$ et en utilisant (2.1.3) on obtient

$$
\|v\|_{H^{1}(Y)} \leq C^{\prime}\left(\|Q v\|_{L^{2}(X)}+\|B v\|_{L^{2}(\partial X)}+\left\|\left.v\right|_{\Gamma_{s_{0}}}\right\|_{L^{2}(\partial X)}\right)^{\mu^{2}}\|v\|_{H^{1}(X)}^{1-\mu^{2}}
$$

ce qui implique (2.1.2).

- Pour démontrer la Proposition 2.1.3, nous allons suivre essentiellement la méthode de Robbiano [29].

\subsection{Inégalité de Carleman au bord}

Soient $x^{\prime}=\left(x_{0}, x^{\prime \prime}\right), x=\left(x^{\prime}, x_{n}\right), \mathbb{R}_{+}^{n+1}=\left\{\left(x^{\prime}, x_{n}\right) \in \mathbb{R}^{n+1} ; x_{n} \geq 0\right\}$ et $K=\left\{x \in \mathbb{R}_{+}^{n+1} ;|x| \leq r_{0}\right\}$. On note $C_{K}^{\infty}\left(\mathbb{R}_{+}^{n+1}\right)$ l'espace des fonctions $C^{\infty}$ de $\mathbb{R}_{+}^{n+1}$ à support dans $K$ et pour $f \in C_{K}^{\infty}\left(\mathbb{R}_{+}^{n+1}\right), f_{0}=\left.f\right|_{x_{n}=0}$.

Soit

$$
\widetilde{Q}=D_{x_{n}}^{2}+R\left(x, D_{x^{\prime}}\right)
$$

avec $D_{x_{j}}=\frac{1}{i} \partial_{x_{j}}$, un opérateur différentiel du second ordre à coefficients $C^{\infty}$ au voisinage de $K$, on note $r\left(x, \xi^{\prime}\right)$ le symbole principal de $R$ et on suppose

$$
r(x, \xi) \in \mathbb{R} \text { et } \exists c>0, \forall\left(x, \xi^{\prime}\right) \in K \times \mathbb{R}^{n} ; \quad r(x, \xi) \geq c\left|\xi^{\prime}\right|^{2} .
$$

Soit $\varphi(x)$ une fonction $C^{\infty}$ réelle définie au voisinage de $K$ et $\gamma$ un paramètre assez grand.

On note

$$
q_{\varphi}(x, \xi)=\left(\xi_{n}+i \gamma \varphi_{x_{n}}^{\prime}\right)^{2}+r\left(x, \xi^{\prime}+i \gamma \varphi_{x^{\prime}}^{\prime}\right)
$$

et on suppose que la fonction $\varphi$ vérifie

$$
\forall x \in K ; \quad \varphi_{x_{n}}^{\prime}(x) \neq 0
$$

et l'hypothèse d'hypoellipticité de Hörmander

$$
\forall(x, \xi) \in K \times \mathbb{R}^{n+1} ; \quad q_{\varphi}(x, \xi)=0 \Longrightarrow\left\{\operatorname{Re} q_{\varphi}, \frac{1}{\gamma} \operatorname{Im} q_{\varphi}\right\}(x, \xi)>0 .
$$

On pose $(f \mid g)=\int_{\mathbb{R}_{+}^{n+1}} f \bar{g} \mathrm{~d} x,\|f\|_{0}^{2}=(f \mid f),\left(f_{0} \mid g_{0}\right)_{0}=\int_{\mathbb{R}^{n}} f_{0} \overline{g_{0}} \mathrm{~d} x^{\prime},\left\|f_{0}\right\|_{0}^{2}=\left(f_{0} \mid f_{0}\right)_{0},\|f\|_{1}^{2}=\gamma^{2}\|f\|_{0}^{2}+$ $\sum_{j=0}^{n}\left\|\partial_{x_{j}} f\right\|_{0}^{2}$ et pour $s \in \mathbb{R}|f|_{s}=\left\|\Lambda^{s} f\right\|_{0}$, où $\Lambda^{s}$ a pour symbole $\left(\gamma^{2}+\left|\xi^{\prime}\right|^{2}\right)^{\frac{s}{2}}$.

Proposition 2.2.1. Soit $\varphi$ vérifiant (2.2.4) et (2.2.5). Il existe $C>0$ et $\gamma_{1}>0$ tels que pour tout $\gamma \geq \gamma_{1}$ et tout $f \in C_{K}^{\infty}\left(\mathbb{R}_{+}^{n+1}\right)$, on ait

$$
\gamma^{-2}\left\|\mathrm{e}^{\gamma \varphi} \widetilde{Q} f\right\|_{0}^{2}+\gamma\left\|\mathrm{e}^{\gamma \varphi} f_{0}\right\|_{0}^{2}+\gamma^{-1}\left\|\left.\mathrm{e}^{\gamma \varphi} \partial_{x_{n}} f\right|_{x_{n}=0}\right\|_{0}^{2} \geq C \gamma\left\|\mathrm{e}^{\gamma \varphi} f\right\|_{0}^{2} .
$$

Remarque 2.2.2. Une estimation de ce type avec $\left\|\mathrm{e}^{\gamma \varphi} f_{0}\right\|_{1}^{2}$ à la place de $\left\|\mathrm{e}^{\gamma \varphi} f_{0}\right\|_{0}^{2}$ a fait l'objet du travail de [18]. Pour le problème qui nous intéresse, nous devons obtenir une estimation avec $f_{0} \in L^{2}$. C'est ce que nous montrons dans la suite. 
Proposition 2.2.3. Soit $\varphi$ vérifiant $(2.2 .5)$ et $\partial_{x_{n}} \varphi\left(x^{\prime}, 0\right)>0$ pour tout $\left(x^{\prime}, 0\right) \in K$. Alors il existe $C>0$ et $\gamma_{1}>0$ tels que pour tout $\gamma \geq \gamma_{1}$ et tout $f \in C_{K}^{\infty}\left(\mathbb{R}_{+}^{n+1}\right)$, on ait

$$
\left\|\mathrm{e}^{\gamma \varphi} \widetilde{Q} f\right\|_{0}^{2}+\gamma\left\|\left.\mathrm{e}^{\gamma \varphi} \partial_{x_{n}} f\right|_{x_{n}=0}\right\|_{0}^{2} \geq C \gamma\left(\left\|\mathrm{e}^{\gamma \varphi} f\right\|_{1}^{2}+\left\|\mathrm{e}^{\gamma \varphi} f_{0}\right\|_{1}^{2}\right) .
$$

Proposition 2.2.4. Soit $\varphi$ vérifiant $(2.2 .5)$ et $\partial_{x_{n}} \varphi\left(x^{\prime}, 0\right)>0$ pour tout $\left(x^{\prime}, 0\right) \in K$. Il existe $C>0$ et $\gamma_{1}>0$ tels que pour tout $\gamma \geq \gamma_{1}$ et tout $f \in C_{K}^{\infty}\left(\mathbb{R}_{+}^{n+1}\right)$, vérifiant $f_{0}=\left.f\right|_{x_{n}=0}=0$ on ait

$$
\left\|\mathrm{e}^{\gamma \varphi} Q f\right\|_{0}^{2} \geq C \gamma\left\|\mathrm{e}^{\gamma \varphi} f\right\|_{1}^{2} .
$$

- Les trois propositions ci-dessus se démontrent classiquement en conjuguant $Q$ par la fonction $\mathrm{e}^{\gamma \varphi}$; plus précisément posons

$$
Q_{\varphi}=\mathrm{e}^{\gamma \varphi} \circ \widetilde{Q} \circ \mathrm{e}^{-\gamma \varphi} .
$$

Proposition 2.2.5. Soit $\varphi$ vérifiant (2.2.4) et (2.2.5). Il existe $C>0$ et $\gamma_{1}>0$ tels que pour tout $\gamma \geq \gamma_{1}$ et tout $h \in C_{K}^{\infty}\left(\mathbb{R}_{+}^{n+1}\right)$, on ait

$$
\gamma^{-2}\left\|Q_{\varphi} h\right\|_{0}^{2}+\gamma\left\|\left.h\right|_{x_{n}=0}\right\|_{0}^{2}+\gamma^{-1}\left\|\left.\partial_{x_{n}} h\right|_{x_{n}=0}\right\|_{0}^{2} \geq C \gamma\|h\|_{0}^{2} .
$$

Proposition 2.2.6. Soit $\varphi$ vérifiant $(2.2 .5)$ et $\partial_{x_{n}} \varphi\left(x^{\prime}, 0\right)>0$ pour tout $\left(x^{\prime}, 0\right) \in K$. Alors il existe $C>0$ et $\gamma_{1}>0$ tels que pour tout $\gamma \geq \gamma_{1}$ et tout $h \in C_{K}^{\infty}\left(\mathbb{R}_{+}^{n+1}\right)$, on ait

$$
\left\|Q_{\varphi} h\right\|_{0}^{2}+\gamma\left\|\left.\left(\partial_{x_{n}}-\gamma \varphi_{x_{n}}^{\prime}\right) h\right|_{x_{n}=0}\right\|_{0}^{2} \geq C \gamma\left(\|h\|_{1}^{2}+\left\|\left.h\right|_{x_{n}=0}\right\|_{1}^{2}\right) .
$$

Proposition 2.2.7. Soit $\varphi$ vérifiant $(2.2 .5)$ et $\partial_{x_{n}} \varphi\left(x^{\prime}, 0\right)>0$ pour tout $\left(x^{\prime}, 0\right) \in K$. Il existe $C>0$ et $\gamma_{1}>0$ tels que pour tout $\gamma \geq \gamma_{1}$ et tout $h \in C_{K}^{\infty}\left(\mathbb{R}_{+}^{n+1}\right)$ vérifiant $h_{0}=\left.h\right|_{x_{n}=0}=0$, on ait

$$
\left\|Q_{\varphi} h\right\|_{0}^{2} \geq C \gamma\|h\|_{1}^{2}
$$

Remarque 2.2.8. Montrons tout d'abord que la condition (2.2.5) peut toujours être réalisée, quitte à changer la fonction $\varphi$ mais en gardant les même lignes de niveau $\varphi=c s t e$.

Soit $\psi$ une fonction $C^{\infty}$ à support compact dont la dérivée ne s'annule jamais et $g$ une fonction croissante, on définit $\varphi$ par $\varphi(x)=g(\psi(x))$, on a

$$
q_{\varphi}(x, \xi)=q(x, \xi)-q\left(x, \gamma \varphi_{x}^{\prime}\right)+2 i \widetilde{q}\left(x, \xi, \gamma \varphi_{x}^{\prime}\right)
$$

où $q(x, \xi)$ est la forme quadratique suivante :

$$
q(x, \xi)=\sum_{1 \leq j, k \leq n} a_{j, k}(x) \xi_{j} \xi_{k}
$$

et $\widetilde{q}(x, \xi, \zeta)$ la forme bilinéaire symétrique associée à la forme quadratique $q(x, \xi)$, on a

$$
\begin{aligned}
\left\{\operatorname{Re} q_{\varphi}, \frac{\operatorname{Im}}{\gamma} q_{\varphi}\right\}= & \partial_{\xi} \operatorname{Re} q_{\varphi} \partial_{x} \frac{\operatorname{Im}}{\gamma} q_{\varphi}-\partial_{\xi} \frac{\operatorname{Im}}{\gamma} q_{\varphi} \partial_{x} \operatorname{Re} q_{\varphi} \\
= & \frac{\operatorname{Im}}{\gamma}\left[\partial_{\xi} q\left(x, \xi-i \gamma \varphi_{x}^{\prime}\right) \partial_{x} q\left(x, \xi+i \gamma \varphi_{x}^{\prime}\right)\right] \\
& +{ }^{t}\left[\partial_{\xi} q\left(x, \xi-i \gamma \varphi_{x}^{\prime}\right)\right]\left(\varphi_{x}^{\prime \prime}\right)\left(\partial_{\xi} q\left(x, \xi+i \gamma \varphi_{x}^{\prime}\right)\right) .
\end{aligned}
$$


Si on remplace $\varphi$ par $\varphi=g(\psi)$ avec $g$ strictement croissante, on obtient en notant $\xi=g^{\prime}(\psi) \eta$ :

$$
\left\{\operatorname{Re} q_{\varphi}, \frac{\operatorname{Im}}{\gamma} q_{\varphi}\right\}(x, \xi)=\left|g^{\prime}(\psi)\right|^{3}\left[\left\{\operatorname{Re} q_{\psi}, \frac{\operatorname{Im}}{\gamma} q_{\psi}\right\}(x, \eta)+\frac{g^{\prime \prime}(\psi)}{g^{\prime}(\psi)} \mid \psi^{\prime}(x) \partial_{\eta} q\left(x, \eta+\left.i \gamma \psi^{\prime}(x)\right|^{2}\right]\right.
$$

et

$$
\mid \psi^{\prime}(x) \partial_{\eta} q\left(x, \eta+\left.i \gamma \psi^{\prime}(x)\right|^{2}=4\left[\gamma^{2}\left|q\left(x, \psi^{\prime}\right)\right|^{2}+\left|\widetilde{q}\left(x, \eta, \psi^{\prime}\right)\right|^{2}\right]\right.
$$

on a

$$
q_{\varphi}=0 \Longleftrightarrow q\left(x, \eta+i \gamma \psi^{\prime}\right)=0 .
$$

- Si $\gamma=0$, on déduit que $q(x, \xi)=0$, ce qui n'est pas vrai car pour tout $(x, \xi) \in K \times \mathbb{R}^{n}, q(x, \xi) \geq C|\xi|^{2}$.

- Si $\gamma \neq 0$, on a $\widetilde{q}\left(x, \eta, \psi^{\prime}\right)=0$ donc $\mid \psi^{\prime}(x) \partial_{\eta} q\left(x, \eta+\left.i \gamma \psi^{\prime}(x)\right|^{2}=4 \gamma^{2}\left|q\left(x, \psi^{\prime}\right)\right|^{2}>0\right.$; prenons $g(z)=\mathrm{e}^{\beta z}$, on a $\frac{g^{\prime \prime}(z)}{g^{\prime}(z)}=\beta$, le terme $\left\{\operatorname{Re} q_{\psi}, \frac{\operatorname{Im}}{\gamma} q_{\psi}\right\}(x, \eta)$ est borné par $\operatorname{Cst}\left(\gamma^{2}+\eta^{2}\right)$ en prenant $\beta$ assez grand, on réalise alors la condition (2.2.5) pour $\varphi=\mathrm{e}^{\beta \psi}$.

Preuves des Propositions 2.2.5, 2.2.6 et 2.2.7. Nous commençons par introduire des notations et rappeler quelques formules.

On note $\mathcal{S}^{j}$ l'espace des symboles tangentiels $a\left(x, \xi^{\prime}, \gamma\right), C^{\infty}$ en $x$ au voisinage de $K, \xi^{\prime} \in \mathbb{R}^{n}$ définis pour $\gamma>0$ et vérifiant :

$$
\forall \alpha, \beta, \exists C_{\alpha, \beta}, \forall x, \xi^{\prime}, \gamma,\left|\partial_{x}^{\alpha} \partial_{\xi^{\prime}}^{\beta} a\right| \leq C_{\alpha, \beta}\left(\gamma+\left|\xi^{\prime}\right|\right)^{j-|\beta|} .
$$

On notera $\mathcal{E}^{j}$ l'espace des opérateurs pseudo-différentiels tangentiels $A=o p(a), a \in S^{j}$, définis par :

$$
(A)(f)(x)=(2 \pi)^{-n} \int \mathrm{e}^{i\left(x^{\prime}-y^{\prime}\right) \xi^{\prime}} a\left(x, \xi^{\prime}, \gamma\right) f\left(y^{\prime}, x_{n}\right) \mathrm{d} y^{\prime} \mathrm{d} \xi^{\prime} .
$$

Nous utiliserons l'inégalité de Gärding suivante (déduite de l'inégalité usuelle) : Soit $A \in \mathcal{E}^{j}$, s'il existe un $c_{0}>0$ tel que $\sigma(A)\left(x, \xi^{\prime}, \gamma\right) \geq c_{0}\left(\gamma^{2}+\left|\xi^{\prime}\right|^{2}\right)$, pour tout $\left(x, \xi^{\prime}\right) \in K \times \mathbb{R}^{n}$, alors

$$
\exists C_{1}>0, \gamma_{1}>0, \forall f \in C_{K}^{\infty}\left(\mathbb{R}_{+}^{n+1}\right), \forall \gamma \geq \gamma_{1} ; \operatorname{Re}(A f \mid f) \geq C_{1}|f|_{1}^{2} .
$$

Enfin, on note $\mathcal{D}^{j}$ le sous-espace de $\mathcal{E}^{j}$ constitué des $A=o p(a)$ où $a\left(x, \xi^{\prime}, \gamma\right)$ est polynomial en $\xi^{\prime}, \gamma$ de degré $j$.

On a, avec $\widetilde{Q}_{2}=\frac{Q_{\varphi}+Q_{\varphi}^{*}}{2}, \quad \widetilde{Q}_{1}=\frac{Q_{\varphi}-Q_{\varphi}^{*}}{2 i \gamma}$

$$
\left\{\begin{array}{l}
Q_{\varphi}=\widetilde{Q}_{2}+i \gamma \widetilde{Q}_{1} \\
\widetilde{Q}_{2}=D_{n}^{2}+Q_{2}, \quad \widetilde{Q}_{1}=D_{n} \varphi_{x_{n}}^{\prime}+\varphi_{x_{n}}^{\prime} D_{n}+2 Q_{1}
\end{array}\right.
$$

où $Q_{1} \in \mathcal{D}^{1}, Q_{2} \in \mathcal{D}^{2}$ ont pour symbole principal

$$
\left\{\begin{array}{l}
q_{2}=\sigma\left(Q_{2}\right)=-\gamma^{2}\left(\varphi_{x_{n}}^{\prime}\right)^{2}+r\left(x, \xi^{\prime}\right)-r\left(x, \gamma \varphi_{x^{\prime}}^{\prime}\right) \\
q_{1}=\sigma\left(Q_{1}\right)=\widetilde{r}\left(x, \xi^{\prime}, \varphi_{x^{\prime}}^{\prime}\right)
\end{array}\right.
$$

où on a noté $\widetilde{r}\left(x, \xi^{\prime}, \eta^{\prime}\right)$ la forme bilinéaire symétrique en $\xi^{\prime}, \eta^{\prime}$ associée à la forme quadratique réelle $r\left(x, \xi^{\prime}\right)$. 
Pour $f, g \in C_{K}^{\infty}\left(\mathbb{R}_{+}^{n+1}\right)$, on posera $\left(f_{0} \mid g_{0}\right)_{0}=\int_{\mathbb{R}^{n}} f_{0} \overline{g_{0}} \mathrm{~d} x^{\prime}$, les formules d'intégration par parties s'écrivent, les opérateurs $\widetilde{Q}_{k}, k=1,2$ étant auto-adjoints par construction ${ }^{3}$,

$$
\begin{gathered}
\left\{\begin{array}{c}
\left(f \mid \widetilde{Q}_{2} g\right)=\left(\widetilde{Q}_{2} f \mid g\right)-i \gamma\left[\left(f \mid D_{n} g\right)_{0}+\left(D_{n} f \mid g\right)_{0}\right] \\
\left(f \mid \widetilde{Q}_{1} g\right)=\left(\widetilde{Q}_{1} f \mid g\right)-2 i \gamma\left(\varphi_{x_{n}}^{\prime} f \mid g\right)_{0}
\end{array}\right. \\
\left\|Q_{\varphi} g\right\|_{0}^{2}=\left\|\widetilde{Q}_{2} g\right\|_{0}^{2}+\gamma^{2}\left\|\widetilde{Q}_{1} g\right\|_{0}^{2}+i \gamma\left[\left(\widetilde{Q}_{1} g \mid \widetilde{Q}_{2} g\right)-\left(\widetilde{Q}_{2} g \mid \widetilde{Q}_{1} g\right)\right]
\end{gathered}
$$

d'où, en utilisant (2.2.9), (2.2.10) et (2.2.11), (2.2.12), devient :

$$
\left\{\begin{array}{l}
\left\|Q_{\varphi} g\right\|_{0}^{2}=\left\|\widetilde{Q}_{2} g\right\|_{0}^{2}+\gamma^{2}\left\|\widetilde{Q}_{1} g\right\|_{0}^{2}+i \gamma\left(\left[\widetilde{Q}_{2}, \widetilde{Q}_{1}\right] g \mid g\right)+\gamma \beta(g) \\
\beta(g)=\left(\widetilde{Q}_{1} g \mid D_{n} g\right)_{0}+\left(\left(D_{n} \widetilde{Q}_{1}-2 \varphi_{x_{n}}^{\prime} \widetilde{Q}_{2}\right) g \mid g\right)_{0}
\end{array}\right.
$$

Par ailleurs on a $i\left[\widetilde{Q}_{2}, \widetilde{Q}_{1}\right] \in\left(\mathcal{D}^{2}+\mathcal{D}^{1} D_{n}+\mathcal{D}^{0} D_{n}^{2}\right)$ et puisque $\varphi_{x_{n}}^{\prime}(x) \neq 0$ pour $x \in K, D_{n}-\frac{1}{2 \varphi_{x_{n}}^{\prime}} \widetilde{Q_{1}} \in \mathcal{D}^{1}$ et $D_{n}^{2}-\widetilde{Q_{2}} \in \mathcal{D}^{2}$ d'où

$$
i\left[\widetilde{Q}_{2}, \widetilde{Q}_{1}\right]=B_{0} \widetilde{Q}_{2}+B_{1} \widetilde{Q}_{1}+B_{2} ; B_{j} \in \mathcal{D}^{j} .
$$

- On rappelle que $\Lambda^{k}=\left(\gamma^{2}+D_{x^{\prime}}^{2}\right)^{\frac{k}{2}} ; \Lambda^{k} \in \mathcal{E}^{k}$.

Lemme 2.2.9. Il existe une constante $c>0$ telle que pour tout $R^{k}$ dans $\mathcal{E}^{k}$ et $g \in C_{k}^{\infty}\left(\mathbb{R}_{+}^{n+1}\right)$ on ait :

$$
\left\|D_{n}^{2} R^{k} g\right\|_{0}^{2} \leq c\left\|D_{n}^{2} \Lambda^{k} g\right\|_{0}^{2}+c\left\|D_{n} \Lambda^{k} g\right\|_{0}^{2}+c\left\|\Lambda^{k} g\right\|_{0}^{2} .
$$

Lemme 2.2.10. Soit $\varphi$ vérifiant (2.2.4) et (2.2.5). Il existe $C>0, \gamma_{1}>0$ tels que pour tout $\gamma \geq \gamma_{1}$ et $g \in C_{k}^{\infty}\left(\mathbb{R}_{+}^{n+1}\right)$ on ait :

$$
\left\|Q_{\varphi} g\right\|_{0}^{2}+\gamma\left|g_{\mid x_{n}=0}\right|_{1}^{2}+\gamma\left|\partial_{x_{n}} g_{\mid x_{n}=0}\right|_{0}^{2} \geq C \gamma\|g\|_{1}^{2}+\gamma\left\|D_{n}^{2} \Lambda^{-1} g\right\|_{0}^{2} .
$$

Remarque 2.2.11. Le Lemme 2.2 .10 est classique sans le second terme à droite $\left(\gamma\left\|D_{n}^{2} \Lambda^{-1} g\right\|_{0}^{2}\right)$. L'essentiel de la preuve consiste à prouver qu'on peut aussi majorer ce terme.

Preuve de la Proposition 2.2.5. Soit $\chi \in C_{K}^{\infty}\left(\mathbb{R}_{+}^{n+1}\right)$ une fonction de troncature, $\chi=1$ sur le support de $h$ et $g=\chi \Lambda^{-1} h \in C_{K}^{\infty}\left(\mathbb{R}_{+}^{n+1}\right)$. En appliquant $Q_{\varphi}$ à $g$ on obtient

$$
\begin{aligned}
Q_{\varphi} g= & \Lambda^{-1} \chi Q_{\varphi} h+\left[\chi, \Lambda^{-1}\right] Q_{\varphi} h+\left[Q_{\varphi}, \Lambda^{-1}\right] \chi h \\
& -\left[\chi, \Lambda^{-1}\right] D_{n}^{2} h+\Lambda^{-1}\left[Q_{\varphi}, \chi\right] h+D_{n}^{2}\left[\chi, \Lambda^{-1}\right] h+T h
\end{aligned}
$$

où

$$
\begin{aligned}
T= & -\left[\left(\gamma \varphi_{x_{n}}^{\prime}\right)^{2},\left[\chi, \Lambda^{-1}\right]\right]+\left[\left(R\left(x, D_{x^{\prime}}\right),\left[\chi, \Lambda^{-1}\right]\right]-\left[\left(R\left(x, \gamma \varphi_{x^{\prime}}^{\prime}\right),\left[\chi, \Lambda^{-1}\right]\right]\right.\right. \\
& +\left[\left(2 \varphi_{x_{n}}^{\prime} D_{n},\left[\chi, \Lambda^{-1}\right]\right]-\left[\varphi_{x_{n}}^{\prime \prime},\left[\chi, \Lambda^{-1}\right]\right]+\left[\widetilde{R}\left(x, D_{x^{\prime}}, \gamma \varphi_{x^{\prime}}^{\prime}\right),\left[\chi, \Lambda^{-1}\right]\right] .\right.
\end{aligned}
$$

En utilisant (2.2.16) et le Lemme 2.2.9, on a

$$
\left\|Q_{\varphi} g\right\|_{0}^{2} \leq c \gamma^{-2}\left\|Q_{\varphi} h\right\|_{0}^{2}+\frac{c}{\gamma^{2}}\|h\|_{0}^{2}+\left\|D_{n}^{2} \Lambda^{-2} h\right\|_{0}^{2}+\left\|D_{n} \Lambda^{-2} h\right\|_{0}^{2}
$$

\footnotetext{
${ }^{3} \widetilde{Q}_{k}^{*} ; k=1,2$ représente l'adjoint formel.
} 
comme

$$
\begin{aligned}
\partial_{x_{j}} g & =\Lambda^{-1} \chi_{x_{j}}^{\prime} h+\left[\chi_{x_{j}}^{\prime}, \Lambda^{-1}\right] h+\Lambda^{-1} \chi \partial_{x_{j}} h \\
& =\chi_{x_{j}}^{\prime} \Lambda^{-1} h+\chi \Lambda^{-1} \partial_{x_{j}} h
\end{aligned}
$$

d'où

$$
\left.\gamma|g|_{x_{n}=0}\right|_{1} ^{2} \leq c \gamma\left\|\left.h\right|_{x_{n}=0}\right\|_{0}^{2}
$$

et

$$
\gamma\left\|\left.\partial_{x_{n}} g\right|_{x_{n}=0}\right\|_{0}^{2} \leq \gamma^{-1}\left\|\left.\partial_{x_{n}} h\right|_{x_{n}=0}\right\|_{0}^{2} .
$$

Comme $\|g\|_{1}^{2}=\|\Lambda g\|_{0}^{2}+\left\|\partial_{x_{n}} g\right\|_{0}^{2}$, on écrit $\Lambda g=\chi h+\Lambda\left[\chi, \Lambda^{-1}\right] h$, ceci implique que $h=\Lambda g-\Lambda\left[\chi, \Lambda^{-1}\right] h \Lambda \Lambda^{-1} h$, on a alors

$$
\|h\|_{0} \leq c\|\Lambda g\|_{0}+c\left\|\Lambda^{-1} h\right\|_{0}
$$

et

$$
\|\Lambda g\|_{0} \geq c\|h\|_{0}-\frac{c}{\gamma}\|h\|_{0}
$$

c'est-à-dire, si $\gamma$ assez grand,

$$
\|\Lambda g\|_{0}^{2} \geq c\|h\|_{0}^{2} .
$$

Par ailleurs, on a d'une part $\partial_{x_{n}} g=\chi_{x_{n}}^{\prime} \Lambda^{-1} h+\chi \partial_{x_{n}} \Lambda^{-1} h$ d'où

$$
\left\|\chi \partial_{x_{n}} \Lambda^{-1} h\right\|_{0}^{2} \leq c\left\|\partial_{x_{n}} g\right\|_{0}^{2}+\frac{1}{\gamma^{2}}\|h\|_{0}^{2}
$$

c'est-à-dire

$$
\left\|\partial_{x_{n}} g\right\|_{0}^{2} \geq c\left\|D_{n} \Lambda^{-1} h\right\|_{0}^{2}-\frac{1}{\gamma^{2}}\|h\|_{0}^{2}+\left\|\chi \partial_{x_{n}} \Lambda^{-1} h\right\|_{0}^{2},
$$

et d'autre part

$$
\begin{aligned}
\gamma\left\|D_{n}^{2} \Lambda^{-1} g\right\|_{0}^{2} & =\gamma\left\|D_{n}^{2} \Lambda^{-1} \chi \Lambda^{-1} h\right\|_{0}^{2} \\
& \geq \gamma\left\|D_{n}^{2} \Lambda^{-2} \chi h\right\|_{0}^{2}-\gamma\left\|D_{n}^{2} \Lambda^{-1}\left[\chi, \Lambda^{-1}\right] h\right\|_{0}^{2}
\end{aligned}
$$

D'après le Lemme 2.2.9 on obtient :

$$
\begin{aligned}
\gamma\left\|D_{n}^{2} \Lambda^{-1} g\right\|_{0}^{2} \geq & \gamma\left\|D_{n}^{2} \Lambda^{-2} h\right\|_{0}^{2}-C \gamma^{-1}\left\|D_{n}^{2} \Lambda^{-2} h\right\|_{0}^{2} \\
& -C \gamma^{-3}\left\|D_{n} \Lambda^{-1} h\right\|_{0}^{2}-C \gamma^{-5}\|h\|_{0}^{2}
\end{aligned}
$$

En utilisant le Lemme 2.2.10 et (2.2.16)-(2.2.22), on obtient le résultat.

Preuve de la Proposition 2.2.6. La Proposition 2.2.6 est un cas particulier de la Proposition 2, p. 247, de [19].

Lemme 2.2.12. Il existe $C>0, \gamma_{0}>0$ tels que pour tout $\gamma \geq \gamma_{0}$ et $g \in C_{k}^{\infty}\left(\mathbb{R}_{+}^{n+1}\right)$ on ait

$$
\gamma\left\|D_{n}^{2} \Lambda^{-1} g\right\|_{0}^{2} \leq C\left\|\widetilde{Q}_{2} g\right\|_{0}^{2}+C \gamma\|g\|_{1}^{2}
$$

Lemme 2.2.13. Il existe $C_{1}>0$ (grand) et $c_{0}>0$ tels que pour tout $\left(x, \xi^{\prime}\right) \in K \times \mathbb{R}^{n}$ on ait

$$
\frac{C_{1}}{\gamma^{2}+\left|\xi^{\prime}\right|^{2}}\left[q_{1}^{2}+\left(\varphi_{x_{n}}^{\prime}\right)^{2} q_{2}\right]^{2}+\sigma\left(B_{2}\right) \geq c_{0}\left(\gamma^{2}+\left|\xi^{\prime}\right|^{2}\right)
$$


Lemme 2.2.14. Il existe $C_{1}>0, \gamma_{1}>0, F_{0} \in \mathcal{E}^{0}$ et $F_{1} \in \mathcal{D}^{1}$ tels que pour tout $\gamma \geq \gamma_{1}$ et $g \in C_{k}^{\infty}\left(\mathbb{R}_{+}^{n+1}\right)$ on ait

$$
\left\|Q_{\varphi} g\right\|_{0}^{2} \geq C_{1} \gamma\|g\|_{1}^{2}+\gamma\left\|D_{n}^{2} \Lambda^{-1} g\right\|_{0}^{2}+\operatorname{Re}\left[\gamma \beta(g)+\gamma\left(D_{n} g+F_{1} \mid F_{0} g\right)_{0}\right] .
$$

Preuve de la Proposition 2.2.7. Si $g=\mathrm{e}^{\gamma \varphi} h$, on a $h_{0}=0$ et l'inégalité de la Proposition 2.2.7 résulte du Lemme 2.2.14 puisque le terme de bord dans l'inégalité du Lemme 2.2.14 vaut alors $2 \int_{\mathbb{R}^{n}} \varphi_{x_{n}}^{\prime}\left|D_{n} g\right|_{0}^{2} \geq 0$ grâce à l'hypothèse $\varphi_{x_{n}}^{\prime}\left(x^{\prime}, 0\right)>0, \forall\left(x^{\prime}, 0\right) \in K$ et $(2.2 .13)$.

Preuve du Lemme 2.2.9. On a

$$
\begin{aligned}
D_{n}^{2} R^{k} g= & D_{n}^{2} R^{k} \Lambda^{-k} \Lambda^{k} g \\
= & D_{n}\left(R^{k} D_{n} \Lambda^{-k} \Lambda^{k} g\right)+D_{n}\left(\left[D_{n}, R^{k}\right] \Lambda^{-k} \Lambda^{k}\right) g \\
= & \left.R^{k} D_{n}^{2} \Lambda^{-k} \Lambda^{k} g+\left[D_{n}, R^{k}\right] D_{n} \Lambda^{-k} \Lambda^{k} g+\left[D_{n}, R^{k}\right] D_{n} \Lambda^{-k} \Lambda^{k}\right) g \\
& +\left[D_{n},\left[D_{n}, R^{k}\right]\right] \Lambda^{-k} \Lambda^{k} g .
\end{aligned}
$$

Puisque $D_{n}^{2} \Lambda^{-k}=\Lambda^{-k} D_{n}^{2}$, on a alors

$$
\begin{aligned}
D_{n}^{2} R^{k} g= & R^{k} \Lambda^{-k} D_{n}^{2} \Lambda^{k} g+\left[D_{n}, R^{k}\right] \Lambda^{-k} D_{n} \Lambda^{k} g+\left[D_{n}, R^{k}\right] \Lambda^{-k} D_{n} \Lambda^{k} g \\
& +\left[D_{n},\left[D_{n}, R^{k}\right]\right] \Lambda^{-k} \Lambda^{k} g
\end{aligned}
$$

et comme on a $R^{k} \Lambda^{-k},\left[D_{n}, R^{k}\right] \Lambda^{-k}$, et $\left[D_{n},\left[D_{n}, R^{k}\right]\right] \Lambda^{-k}$ sont dans $\mathcal{E}^{0}$ (car $R^{k} \in \mathcal{E}^{k}$, ceci implique $\left[D_{n}, R^{k}\right] \in$ $\left.\mathcal{E}^{k}\right)$, donc bornés sur $L^{2}$ d'où le résultat.

Preuve du Lemme 2.2.10. La preuve du Lemme 2.2.10 résulte du Lemme 2.2.14.

Preuve du Lemme 2.2.12. On a d'après (2.2.9) et (2.2.10)

$$
\begin{aligned}
\left\|D_{n}^{2} \Lambda^{-1} g\right\|_{0}^{2} & =\left(D_{n}^{2} \Lambda^{-1} g \mid D_{n}^{2} \Lambda^{-1} g\right) \\
& =\left(D_{n}^{2} \Lambda^{-1} g \mid \widetilde{Q}_{2} \Lambda^{-1} g-Q_{2} \Lambda^{-1} g\right) \\
& =\left(D_{n}^{2} \Lambda^{-1} g \mid \widetilde{Q}_{2} \Lambda^{-1} g\right)+\left(D_{n}^{2} \Lambda^{-1} g \mid\left(\gamma \varphi_{x_{n}}^{\prime}\right)^{2} \Lambda^{-1} g\right) \\
& -\left(D_{n}^{2} \Lambda^{-1} g \mid R \Lambda^{-1} g\right)+\left(D_{n}^{2} \Lambda^{-1} g \mid R\left(x, \gamma \varphi_{x^{\prime}}^{\prime}\right) \Lambda^{-1} g\right) \\
& =t_{1}+t_{2}+t_{3}+t_{4} .
\end{aligned}
$$

On va majorer les termes $t_{j}, j=1, \ldots, 4$ en utilisant l'inégalité de Cauchy-Schwartz

$$
\left|t_{1}\right|=\left|\left(D_{n}^{2} \Lambda^{-1} g \mid \widetilde{Q}_{2} \Lambda^{-1} g\right)\right| \leq C \epsilon\left\|D_{n}^{2} \Lambda^{-1} g\right\|_{0}^{2}+\frac{C}{\epsilon}\left\|\widetilde{Q}_{2} \Lambda^{-1} g\right\|_{0}^{2} .
$$

Comme

$$
\widetilde{Q}_{2} \Lambda^{-1} g=\Lambda^{-1} \widetilde{Q}_{2} g-\left[\left(\gamma \varphi_{x_{n}}^{\prime}\right)^{2}, \Lambda^{-1}\right] g-\left[R\left(x, \gamma \varphi_{x_{n}}^{\prime}\right), \Lambda^{-1}\right] g+\left[R\left(x, D_{x^{\prime}}\right), \Lambda^{-1}\right] g
$$

et $\left[\left(\gamma \varphi_{x_{n}}^{\prime}\right)^{2}, \Lambda^{-1}\right],\left[R\left(x, \gamma \varphi_{x^{\prime}}^{\prime}\right), \Lambda^{-1}\right]$ et $\left[R\left(x, D_{x^{\prime}}\right), \Lambda^{-1}\right]$ sont dans $\mathcal{E}^{0}$ alors

$$
\left|t_{1}\right| \leq C \epsilon\left\|D_{n}^{2} \Lambda^{-1} g\right\|_{0}^{2}+\frac{C}{\epsilon} \gamma^{-2}\left\|\widetilde{Q}_{2} g\right\|_{0}^{2}+\frac{C}{\epsilon}\|g\|_{0}^{2},
$$

$\left|-t_{3}\right|=\left|-\left(D_{n}^{2} \Lambda^{-1} g \mid R\left(x, D_{x^{\prime}}\right) \Lambda^{-1} g\right)\right| \leq C \epsilon\left\|D_{n}^{2} \Lambda^{-1} g\right\|_{0}^{2}+\frac{C}{\epsilon}\left\|R\left(x, D_{x^{\prime}}\right) \Lambda^{-1} g\right\|_{0}^{2} ;$ comme $R\left(x, D_{x^{\prime}}\right) \Lambda^{-1} \in \mathcal{E}^{1}$ alors

$$
\left|-t_{3}\right| \leq C \epsilon\left\|D_{n}^{2} \Lambda^{-1} g\right\|_{0}^{2}+\frac{C}{\epsilon}\|g\|_{1}^{2} .
$$


De même, les termes $\left|t_{2}\right|$ et $\left|t_{4}\right|$ sont majorés par les termes suivants :

$$
C \epsilon\left\|D_{n}^{2} \Lambda^{-1} g\right\|_{0}^{2}+\frac{C}{\epsilon}|g|_{1}^{2} .
$$

D'après (2.2.23)-(2.2.25), on a en prenant $\epsilon$ assez petit

$$
\left\|D_{n}^{2} \Lambda^{-1} g\right\|_{0}^{2} \leq C(\epsilon) \gamma^{-2}\left\|\widetilde{Q}_{2} g\right\|_{0}^{2}+C(\epsilon)|g|_{1}^{2},
$$

on multiplie cette dernière inégalité par $\gamma$, on obtient le résultat.

Preuve du Lemme 2.2.13. On a $q_{\varphi}(x, \xi)=\xi_{n}^{2}+q_{2}+2 i \gamma\left(\xi_{n} \varphi_{x_{n}}^{\prime}+q_{1}\right)$ et l'hypothèse (2.2.5) nous permet de dire que

$$
\forall(x, \xi) \in K \times R^{n+1},\left\{\xi_{n}^{2}+q_{2}=0 \text { et } \xi_{n} \varphi_{x_{n}}^{\prime}+q_{1}=0\right\} \Rightarrow\left\{\xi_{n}^{2}+q_{2}, \xi_{n} \varphi_{x_{n}}^{\prime}+q_{1}\right\}>0 .
$$

D'après (2.2.14) on a

$$
\left\{\xi_{n}^{2}+q_{2}, \xi_{n} \varphi_{x_{n}}^{\prime}+q_{1}\right\}=\sigma\left(B_{0}\right)\left(\xi_{n}^{2}+q_{2}\right)+\sigma\left(B_{1}\right)\left(\xi_{n} \varphi_{x_{n}}^{\prime}+q_{1}\right)+\sigma\left(B_{2}\right) .
$$

Montrons que

$$
\forall\left(x, \xi^{\prime}\right) \in K \times \mathbb{R}^{n}, q_{1}^{2}+\left(\varphi_{x_{n}}^{\prime}\right)^{2} q_{2}=0 \Rightarrow \sigma\left(B_{2}\right)>0 .
$$

En effet, si $q_{1}^{2}+\left(\varphi_{x_{n}}^{\prime}\right)^{2} q_{2}=0$, posons $\xi_{n}=-\frac{q_{1}}{\varphi_{x_{n}}^{\prime}} \in \mathbb{R}$, on a $\xi_{n} \varphi_{x_{n}}^{\prime}+q_{1}=0$ et $\xi_{n}^{2}+q_{2}=0$, (2.2.26) et (2.2.27) entraînent $\sigma\left(B_{2}\right)>0$ :

Montrons tout d'abord que pour tout $x \in K$ et $\Gamma^{2}+\Xi^{2}=1$, on a

$$
\left.\left[C_{1}\left(q_{1}^{2}+\left(\varphi_{x_{n}}^{\prime}\right)^{2} q_{2}\right)^{2}+\sigma\left(B_{2}\right)\right]\right|_{(x, \Gamma, \Xi)} \geq c_{0} .
$$

Par l'absurde, il existe $\left(x_{k}, \Gamma_{k}, \Xi_{k}\right)_{k \in \mathrm{N}} ; x_{k} \in K, \Gamma_{k}^{2}+\Xi_{k}^{2}=1$ tels que

$$
\left.\left[C_{k}\left(q_{1}^{2}+\left(\varphi_{x_{n}}^{\prime}\right)^{2} q_{2}\right)^{2}+\sigma\left(B_{2}\right)\right]\right|_{\left(x_{k}, \Gamma_{k}, \Xi_{k}\right)} \leq \frac{1}{C_{k}}
$$

et on peut supposer, quitte à extraire une sous-suite $x_{k} \rightarrow x, \Gamma_{k} \rightarrow \Gamma, \Xi_{k} \rightarrow \Xi$ et $C_{k} \rightarrow+\infty$ lorsque $k \rightarrow$ $+\infty$ en divisant (2.2.30) par $C_{k}$ et faisant tendre $k$ vers $+\infty$, qu'on a alors $\left.\left(q_{1}^{2}+\left(\varphi_{x_{n}}\right)^{2} q_{2}\right)^{2}\right|_{(x, \Gamma, \Xi)} \leq 0$ d'où $\left.\left(q_{1}^{2}+\left(\varphi_{x_{n}}^{\prime}\right)^{2} q_{2}\right)^{2}\right|_{(x, \Gamma, \Xi)}=0$, on déduit de $(2.2 .28)$

$$
\left.\sigma\left(B_{2}\right)\right|_{(x, \Gamma, \Xi)}>0 .
$$

D'autre part, de $(2.2 .30)$ on a $\left.\sigma\left(B_{2}\right)\right|_{\left(x_{k}, \Gamma_{k}, \Xi_{k}\right)} \leq \frac{1}{C_{k}}$ en faisant tendre $k \rightarrow+\infty$, on a $\left.\sigma\left(B_{2}\right)\right|_{(x, \Gamma, \Xi)} \leq 0$ en contradiction avec (2.2.31), d'où (2.2.29). On obtient le lemme par homogénéité en posant $\Gamma=\frac{\gamma}{\left(\gamma^{2}+\left|\xi^{\prime}\right|^{2}\right)^{\frac{1}{2}}}$ et $\Xi=\frac{\xi^{\prime}}{\left(\gamma^{2}+\left|\xi^{\prime}\right|^{2}\right)^{\frac{1}{2}}}$.

Preuve du Lemme 2.2.14. D'après (2.2.13) et (2.2.14) on a

$$
\left\|Q_{\varphi} g\right\|_{0}^{2}-\operatorname{Re}(\gamma \beta(g))=\left\|\widetilde{Q_{2}} g\right\|_{0}^{2}+\gamma^{2}\left\|\widetilde{Q}_{1} g\right\|_{0}^{2}+\gamma\left(B_{2} g \mid g\right)+\gamma\left(B_{0} \widetilde{Q}_{2} g+B_{1} \widetilde{Q}_{1} g \mid g\right)
$$

et l'inégalité de Cauchy-Schwartz nous donne :

$$
\left|\gamma\left(B_{0} \widetilde{Q}_{2} g \mid g\right)\right| \leq \epsilon . C\left\|\widetilde{Q}_{2} g\right\|_{0}^{2}+C \frac{\gamma^{2}}{\epsilon}\|g\|_{0}^{2}
$$


et

$$
\left|\gamma\left(B_{1} \widetilde{Q}_{1} g \mid g\right)\right|=\left.\left|\left(\sqrt{\gamma} \widetilde{Q}_{1} g \mid \sqrt{\gamma} B_{1}^{*} g\right) \leq C \frac{\gamma}{\epsilon}\left\|\widetilde{Q}_{1} g\right\|_{0}^{2}+C \gamma \cdot \epsilon\right| g\right|_{1} ^{2}
$$

d'où

$$
\left|\gamma\left(B_{0} \widetilde{Q}_{2} g+B_{1} \widetilde{Q}_{1} g \mid g\right)\right| \leq \epsilon . C\left\|\widetilde{Q}_{2} g\right\|_{0}^{2}+\frac{C}{\epsilon} \gamma\left\|\widetilde{Q}_{1} g\right\|_{0}^{2}+C \gamma \cdot \epsilon|g|_{1}^{2}+\frac{C}{\epsilon} \gamma^{2}\|g\|_{0}^{2}
$$

Par ailleurs, d'après l'inégalité de Gärding et le Lemme 2.2.13, il existe $C_{1}>0, \gamma_{1}>0$ tels que pour tout $\gamma \geq \gamma_{1}, g \in C_{K}^{\infty}\left(\mathbb{R}_{+}^{n+1}\right)$ on ait :

$$
\operatorname{Re} \gamma\left(B_{2} g \mid g\right) \geq C_{1} \gamma|g|_{1}^{2}-\gamma \operatorname{Re}\left(\left(Q_{1}^{2}+\left(\varphi_{x_{n}}^{\prime}\right)^{2} Q_{2}\right) g \mid F_{0} g\right)
$$

où $F_{0} \in \mathcal{E}^{0}$ a pour symbole principal :

$$
\sigma\left(F_{0}\right)=\frac{C_{1}}{\gamma^{2}+\left|\xi^{\prime}\right|^{2}}\left(q_{1}^{2}+\left(\varphi_{x_{n}}^{\prime}\right)^{2} q_{2}\right)
$$

D'autre part, on a d'après (2.2.9)

d'où

$$
D_{n}=\frac{\widetilde{Q}_{1}}{2 \varphi_{x_{n}}^{\prime}}-\frac{1}{\varphi_{x_{n}}^{\prime}} Q_{1}-\frac{1}{2 i \varphi_{x_{n}}^{\prime}} \varphi_{x_{n}}^{\prime \prime}
$$

$$
\left\|D_{n} g\right\|_{0} \leq c\left\|\widetilde{Q}_{1} g\right\|_{0}+c\left\|Q_{1} g\right\|_{0}+c\|g\|_{0}
$$

or

$$
\|g\|_{1} \simeq\left\|D_{n} g\right\|_{0}+|g|_{1}
$$

On en déduit alors

$$
\|g\|_{1} \leq c\left(\left\|\widetilde{Q}_{1} g\right\|_{0}+|g|_{1}\right) .
$$

On a également

$$
Q_{1}=\frac{1}{2}\left(\widetilde{Q_{1}}-\left[D_{n}, \varphi_{x_{n}}^{\prime}\right]\right)-\varphi_{x_{n}}^{\prime} D_{n}
$$

d'où

$$
\begin{aligned}
Q_{1}^{2}+\left(\varphi_{x_{n}}^{\prime}\right)^{2} Q_{2}= & \left(\varphi_{x_{n}}^{\prime} D_{n}-\frac{1}{2}\left(\widetilde{Q_{1}}-\left[D_{n}, \varphi_{x_{n}}^{\prime}\right]\right) \varphi_{x_{n}}^{\prime} D_{n}+\frac{Q_{1}}{2}\left(\widetilde{Q_{1}}-\left[D_{n}, \varphi_{x_{n}}^{\prime}\right]\right)\right. \\
& +\left(\varphi_{x_{n}}^{\prime}\right)^{2}\left[\widetilde{Q}_{2}-D_{n}^{2}\right] \\
= & \left(\varphi_{x_{n}}^{\prime}\right)^{2} D_{n}^{2}+\frac{1}{i} \varphi_{x_{n}}^{\prime \prime} \varphi_{x_{n}}^{\prime} D_{n}-\frac{1}{2} \widetilde{Q}_{1} \varphi_{x_{n}}^{\prime} D_{n}+\frac{1}{2}\left[D_{n}, \varphi_{x_{n}}^{\prime}\right] \varphi_{x_{n}}^{\prime} D_{n} \\
& +\frac{1}{2} Q_{1} \widetilde{Q}_{1}-\frac{1}{2} Q_{1}\left[D_{n}, \varphi_{x_{n}}^{\prime}\right]+\left(\varphi_{x_{n}}^{\prime}\right)^{2} \widetilde{Q}_{2}-\left(\varphi_{x_{n}}^{\prime}\right)^{2} D_{n}^{2} .
\end{aligned}
$$

Ceci nous permet de dire que :

$$
Q_{1}^{2}+\left(\varphi_{x_{n}}^{\prime}\right)^{2} Q_{2}=\left(\varphi_{x_{n}}^{\prime}\right)^{2} \widetilde{Q}_{2}-\frac{1}{2} D_{n} \varphi_{x_{n}}^{\prime} \widetilde{Q}_{1}+C_{1} \widetilde{Q}_{1}+C_{0} D_{n}+\widetilde{C}_{1}
$$

où $C_{j}, \widetilde{C}_{j} \in \mathcal{D}^{j}$. On a

$$
\begin{aligned}
\operatorname{Re}\left(\left(Q_{1}^{2}+\left(\varphi_{x_{n}}^{\prime}\right)^{2} Q_{2}\right) g \mid F_{0} g\right)= & \left.\operatorname{Re}\left\{\left(\varphi_{x_{n}}^{\prime}\right)^{2} \widetilde{Q}_{2}\right) g \mid F_{0} g\right)+\left(\frac{1}{2 i} \varphi_{x_{n}}^{\prime} \widetilde{Q}_{1} g \mid F_{0} g\right)_{0} \\
& +\left(\frac{1}{2} \varphi_{x_{n}}^{\prime} \widetilde{Q}_{1} \mid F_{0} D_{n} g\right)+\left(C_{1} \widetilde{Q}_{1} g \mid F_{0} g\right) \\
& \left.+\left(C_{0} D_{n} g \mid F_{0} g\right)+\left(\widetilde{C}_{1} g \mid F_{0} g\right)\right\} .
\end{aligned}
$$


On va majorer chaque terme

$$
\begin{gathered}
\left.\left|\left(\left(\varphi_{x_{n}}^{\prime}\right)^{2} \widetilde{Q}_{2}\right) g\right| F_{0} g\right) \mid \leq \frac{c \epsilon}{\gamma}\left\|\widetilde{Q}_{2} g\right\|_{0}^{2}+\frac{C \gamma}{\epsilon}\|g\|_{0}^{2} \\
\left|\left(C_{1} \widetilde{Q}_{1} g \mid F_{0} g\right)\right| \leq c \epsilon \gamma\left\|\widetilde{Q}_{1} g\right\|_{0}^{2}+\frac{C}{\epsilon \gamma}|g|_{1}^{2} \\
\left|\left(C_{0} D_{n} g \mid F_{0} g\right)\right| \leq \frac{c \epsilon}{\gamma}\left\|D_{n} g\right\|_{0}^{2}+\frac{C \gamma}{\epsilon}\|g\|_{0}^{2} \\
\left.\mid\left(\widetilde{C}_{1} g \mid F_{0} g\right)\right\}\left.\left|\leq \frac{c \epsilon}{\gamma}\right| g\right|_{1} ^{2}+\frac{C \gamma}{\epsilon}\|g\|_{0}^{2} \\
\left|\left(\varphi_{x_{n}}^{\prime} \widetilde{Q}_{1} \mid F_{0} D_{n} g\right)\right| \leq c \epsilon \gamma\left\|\widetilde{Q}_{1} g\right\|_{0}^{2}+\frac{C}{\epsilon \gamma}\left\|D_{n} g\right\|_{0}^{2} .
\end{gathered}
$$

D'après (2.2.36)-(2.2.40) on déduit que :

$$
\begin{aligned}
\operatorname{Re}\left(\left(Q_{1}^{2}+\left(\varphi_{x_{n}}^{\prime}\right)^{2} Q_{2}\right) g \mid F_{0} g\right) \leq & \operatorname{Re}\left(\frac{1}{2 i} \varphi_{x_{n}}^{\prime} \widetilde{Q}_{1} g \mid F_{0} g\right)_{0}+\operatorname{cst}\left(\frac{\epsilon}{\gamma}\left\|\widetilde{Q_{2}} g\right\|_{0}^{2}\right. \\
& \left.+\epsilon \gamma\left\|\widetilde{Q_{1}} g\right\|_{0}^{2}\right)+\frac{C}{\epsilon \gamma}\|g\|_{1}^{2}
\end{aligned}
$$

d'où

$$
\begin{aligned}
-\gamma \operatorname{Re}\left(\left(Q_{1}^{2}+\left(\varphi_{x_{n}}^{\prime}\right)^{2} Q_{2}\right) g \mid F_{0} g\right) \geq & \operatorname{Re}\left(\frac{1}{2 i} \varphi_{x_{n}}^{\prime} \widetilde{Q}_{1} g \mid F_{0} g\right)_{0} \\
& -\operatorname{cst}\left(\epsilon\left\|\widetilde{Q_{2}} g\right\|_{0}^{2}+\epsilon \gamma^{2}\left\|\widetilde{Q_{1}} g\right\|_{0}^{2}+\frac{1}{\epsilon}\|g\|_{1}^{2}\right) .
\end{aligned}
$$

En utilisant (2.2.32)-(2.2.34) et (2.2.41), on trouve, si $\gamma \gg \frac{1}{\epsilon}$,

$$
\begin{aligned}
\left\|Q_{\varphi} g\right\|_{L^{2}}^{2}-\operatorname{Re}(\gamma \beta(g)) \geq & C_{1} \gamma\|g\|_{1}^{2}+c \gamma\|g\|_{1}^{2}+\frac{1}{2}\left\|\widetilde{Q}_{2} g\right\|_{0}^{2} \\
& +\operatorname{Re} \gamma\left(D_{n} g+F_{1} g \mid F_{0} g\right)_{0}
\end{aligned}
$$

où $F_{1} \in \mathcal{D}^{1}$ et $F_{0} \in \mathcal{E}^{0}$; grâce au Lemme 2.2.12 on déduit le résultat.

\subsection{Inégalité d'interpolation locale}

Le but de ce paragraphe est de prouver les inégalités d'interpolation (2.1.3) et (2.1.4). Cela va se décomposer en plusieurs étapes. On va d'abord prouver comme conséquence directe des inégalités de Carleman, des inégalités de type (2.1.3) et (2.1.4) sur des domaines assez petits. Il s'agit ensuite de propager ces inégalités de proche en proche. Pour cela, on va introduire un lemme de récurrence adapté à notre situation. Enfin on fera les constructions géométriques permettant de prouver (2.1.3) et (2.1.4).

Nous noterons $B^{+}(0, r)=\left\{x \in \mathbb{R}^{n},|x| \leq r, x_{n}>0\right\}$.

Lemme 2.3.1. Il existe $\mu \in] 0,1\left[\right.$ et $C>0$ tels que, pour tout $v \in C^{\infty}\left(\mathbb{R}_{+}^{n+1}\right)$, on ait

$$
\begin{aligned}
\|v\|_{L^{2}\left(B^{+}(0, r)\right)} \leq & C\left[\|\widetilde{Q} v\|_{L^{2}\left(B^{+}(0,5 r)\right)}+\|v\|_{L^{2}\left(B^{+}(0,5 r) \cap\left\{x_{n}=0\right\}\right)}\right. \\
& \left.+\left\|\partial_{n} v\right\|_{L^{2}\left(B^{+}(0,5 r) \cap\left\{x_{n}=0\right\}\right)}\right]^{\mu}\left(\|v\|_{H^{1}\left(B^{+}(0,5 r)\right.}\right)^{1-\mu} .
\end{aligned}
$$


Preuve. On pose $r_{1}=\frac{r}{5}, r_{1}^{\prime}=\frac{2 r}{5}, r_{2}=\frac{3 r}{5}<r, r_{2}^{\prime}=\frac{5 r}{2}, r_{3}=3 r, r_{3}^{\prime}=4 r$ de sorte que $r_{1}<r_{1}^{\prime}<r_{2}<r_{2}^{\prime}<r_{3}<$ $r_{3}^{\prime}$ et soit $\psi(s, x)=s^{2}+\left|x^{\prime}\right|^{2}+\left|x_{n}+r\right|^{2}$. On pose

$$
\begin{aligned}
V & =\left\{(s, x) \in X ; r_{1}^{2}<\psi(s, x)<\left(r_{3}^{\prime}\right)^{2}\right\} \\
V_{j} & =\left\{(s, x) \in X ; r_{j}^{2}<\psi(s, x)<\left(r_{j}^{\prime}\right)^{2}\right\} .
\end{aligned}
$$

En vertu de la Proposition 2.2.1 (en divisant l'inégalité de cette proposition par $\gamma$ ), on a pour $\gamma>\gamma_{1}, f \in$ $C_{\bar{V}}^{\infty}\left(\mathbb{R}_{+}^{n+1}\right)$

$$
\begin{aligned}
C\left\|\mathrm{e}^{\gamma \mathrm{e}^{-\beta \psi(s, x)}} f\right\|_{L^{2}(V)} \leq & \left\|\mathrm{e}^{\gamma \mathrm{e}^{-\beta \psi(s, x)}} \widetilde{Q} f\right\|_{L^{2}(V)} \\
& +\left\|\left.\mathrm{e}^{\gamma \mathrm{e}^{-\beta \psi(s, x)}} f\right|_{x_{n}=0}\right\|_{L^{2}\left(\partial V \cap\left\{x_{n}=0\right\}\right)}+\left\|\mathrm{e}^{\gamma \mathrm{e}^{-\beta \psi(s, x)}} \partial_{n} f\right\|_{L^{2}\left(\partial V \cap\left\{x_{n}=0\right\}\right)} .
\end{aligned}
$$

Introduisons la fonction de troncature $\tilde{\chi}$ de $\theta$ définie par

$$
\widetilde{\chi}(\theta)= \begin{cases}1 ; & \frac{4}{25} r^{2} \leq \theta \leq 9 r^{2} \\ 0 ; & \theta \leq \frac{r^{2}}{25} \text { ou } \theta \geq 16 r^{2}\end{cases}
$$

et considérons $\chi \in C_{\bar{V}}^{\infty}\left(\mathbb{R}^{n+1}\right)$ definie par $\chi(s, x)=\tilde{\chi}(\psi(s, x))$, on prend $f=\chi v$ et de (2.3.2), on obtient en écrivant

$$
\widetilde{Q} f=\chi \widetilde{Q} v+[\widetilde{Q}, \chi] v
$$

$\left([\widetilde{Q}, \chi]\right.$ est un opérateur différentiel d'ordre 1 supporté sur $\left.V_{1} \cup V_{3}\right), B^{+}(0, r) \subset V_{2}$ et $V \cap \mathbb{R}_{+}^{n+1} \subset B^{+}(0,5 r)$

$$
\begin{aligned}
C \mathrm{e}^{\gamma \mathrm{e}^{-\beta r_{2}}}\|v\|_{L^{2}\left(B^{+}(0, r)\right) \leq} \leq \mathrm{e}^{\gamma \mathrm{e}^{-\beta r_{2}}}\left[\|\widetilde{Q} v\|_{L^{2}\left(B^{+}(0,5 r)\right)}+\|v\|_{L^{2}\left(B^{+}(0,5 r) \cap\left\{x_{n}=0\right\}\right)}\right. \\
\left.+\left\|\partial_{n} v\right\|_{L^{2}\left(B^{+}(0,5 r) \cap\left\{x_{n}=0\right\}\right)}\right]+\mathrm{e}^{\gamma \mathrm{e}^{-\beta r_{3}}\|v\|_{H^{1}\left(B^{+}(0,5 r)\right)} .}
\end{aligned}
$$

On pose $C_{1}=\mathrm{e}^{-\beta r_{2}^{\prime}}-\mathrm{e}^{-\beta r_{3}}>0$ et $C_{2}=\mathrm{e}^{-\beta r_{2}}-\mathrm{e}^{-\beta r_{2}^{\prime}}>0$, on obtient

$$
\begin{aligned}
\|v\|_{L^{2}\left(B^{+}(0, r)\right)} \leq & C\left(\mathrm { e } ^ { \gamma C _ { 2 } } \left[\|\widetilde{Q} v\|_{L^{2}\left(B^{+}(0,5 r)\right)}+\|v\|_{L^{2}\left(B^{+}(0,5 r) \cap\left\{x_{n}=0\right\}\right)}\right.\right. \\
& \left.\left.+\left\|\partial_{n} v\right\|_{L^{2}\left(B^{+}(0,5 r) \cap\left\{x_{n}=0\right\}\right)}\right]+\mathrm{e}^{-\gamma C_{1}}\|v\|_{H^{1}\left(B^{+}(0,5 r)\right)}\right) .
\end{aligned}
$$

On optimise en $\gamma$ (comme dans [28]), on a alors

$$
\begin{aligned}
\|v\|_{L^{2}\left(B^{+}(0, r)\right)} \leq & C\left(\|\widetilde{Q} v\|_{L^{2}\left(B^{+}(0,5 r)\right)}+\|v\|_{L^{2}\left(B^{+}(0,5 r) \cap\left\{x_{n}=0\right\}\right)}\right. \\
& \left.+\left\|\partial_{n} v\right\|_{L^{2}\left(B^{+}(0,5 r) \cap\left\{x_{n}=0\right\}\right)}\right)^{\frac{C_{1}}{C_{1}+C_{2}}\left(\|v\|_{H^{1}\left(B^{+}(0,5 r)\right)}\right)^{\frac{C_{2}}{C_{1}+C_{2}}}}
\end{aligned}
$$

En posant $\mu=\frac{C_{1}}{C_{1}+C_{2}}$ on obtient le résultat.

Lemme 2.3.2. Il existe $\left.\mu_{0} \in\right] 0,1[$ et $C>0$ tels que, pour tout $\left.\mu \in] 0, \mu_{0}\right]$ et tout $v \in C^{\infty}\left(\mathbb{R}_{+}^{n+1}\right)$, on ait

$$
\|v\|_{H^{1}\left(U_{0}\right)} \leq C\left[\|Q v\|_{L^{2}(X)}+\left\|\left.v\right|_{\Gamma_{s_{0}}}\right\|_{L^{2}(\partial X)}+\|B v\|_{L^{2}(\partial X)}\right]^{\mu}\left(\|v\|_{H^{1}(X)}\right)^{1-\mu}
$$

où $U_{0}, X$ ont été définis à la Section 2.1 .

Preuve. Soit $\left(s_{1}, x_{1}\right) \in \Gamma_{s_{0}}$, il existe alors un voisinage de $\left(s_{1}, x_{1}\right)$ noté $U$ et un difféomorphisme noté $G$ défini de $U \cap X$ vers $V_{(0,0)} \cap\left\{x_{n} \geq 0\right\}$ tels que $G\left(\left(s_{1}, x_{1}\right)\right)=(0,0)$ et $B\left(\left(s_{1}, x_{1}\right), r^{\prime}\right) \cap X \subset G^{-1}\left(B(0, r) \cap\left\{x_{n} \geq 0\right\}\right)$, 
avec $r^{\prime}$ assez petit, $\widetilde{Q}$ (resp. $\partial_{n}$ ) se transforme par $G$ en $Q$ (resp. B) et en utilisant (2.3.1) et le fait que $G$ transforme les normes en normes équivalentes, on a

$$
\|v\|_{L^{2}\left(B^{+}\left(\left(s_{1}, x_{1}\right), r^{\prime}\right)\right)} \leq C\left(\|Q v\|_{L^{2}(X)}+\left\|\left.v\right|_{\Gamma_{s_{0}}}\right\|_{L^{2}(\partial X)}+\|B v\|_{L^{2}(\partial X)}\right)^{\mu} \cdot\left(\|v\|_{H^{1}(X)}\right)^{1-\mu} .
$$

Pour terminer la preuve de l'inégalité (2.3.3), on va rappeler le résultat suivant (dû à l'ellipticité de l'opérateur Q); soit $U_{0}=B\left(\left(s_{2}, x_{2}\right), r^{\prime \prime}\right) \subset B\left(\left(s_{1}, x_{1}\right), r^{\prime}\right) ; U_{0}$ dans l'intérieur de $X$.

Lemme 2.3.3. Il existe $C>0$ tel que, pour tout $v$, on ait

$$
\|v\|_{H^{1}\left(U_{0}\right)} \leq C\left(\|v\|_{L^{2}\left(B\left(\left(s_{1}, x_{1}\right), r^{\prime}\right)\right)}+\|Q v\|_{L^{2}\left(B\left(\left(s_{1}, x_{1}\right), r^{\prime}\right)\right)}\right) .
$$

À partir des inégalités (2.3.4) et (2.3.5,) on a le Lemme 2.3.2, ceci démontre l'inégalité (2.1.3).

Lemme 2.3.4 (de récurrence). Soient $\nu \in] 0,1\left[\right.$ et $\alpha_{k}>0$ vérifiant pour tout $k \geq 0, \alpha_{k} \leq F^{1-\nu}\left(\alpha_{k-1}+E\right)^{\nu}$ et $\alpha_{k} \leq F$ où $E>0, F>0$. Alors pour $\left.\mu \in\right] 0, \nu^{N}[; N>0$, on a

$$
\alpha_{N} \leq 2^{\frac{1}{1-\nu}} F^{1-\mu}\left(\alpha_{0}+E\right)^{\mu} .
$$

Preuve.

- Si $F \leq \alpha_{0}+E$, le résultat est immédiat.

- Si $\alpha_{0}+E \leq F$, en particulier $E \leq F$, on a $\alpha_{k} \leq F^{1-\nu}\left(\alpha_{k-1}+E\right)^{\nu}$ qui implique

$$
\frac{\alpha_{k}}{F} \leq\left(\frac{\alpha_{k-1}+E}{F}\right)^{\nu}
$$

et

$$
\frac{E}{F} \leq\left(\frac{E}{F}\right)^{\nu} \leq\left(\frac{\alpha_{k-1}+E}{F}\right)^{\nu}
$$

De (2.3.6) et (2.3.7) on obtient $\frac{\alpha_{k}+E}{F} \leq\left(2 \frac{\alpha_{k-1}+E}{F}\right)^{\nu}$, ceci implique par itération

$$
\alpha_{N} \leq 2^{\frac{1}{1-\nu}} F^{1-\nu^{N}}\left(\alpha_{0}+E\right)^{\nu^{N}} ;
$$

comme $\alpha_{0}+E \leq F,(2.3 .8)$ implique le lemme pour $\mu \leq \nu^{N}$.

- Estimation sur $X_{\delta}$.

On note $X_{\delta}=\left[-\frac{s_{0}}{2}, \frac{s_{0}}{2}\right] \times \Omega_{\delta}$, où $\delta$ est fixé et $\Omega_{\delta}=\{x \in \Omega ; d(x, \partial \Omega) \geq \delta\}$. Soit $r$ assez petit $(r \ll \delta$ et $\left.r \ll s_{0}\right)$, on recouvre $X_{\delta}$ par un nombre fini de boules de rayon $r$ et de centre $y_{j}=\left(s_{j}, x_{j}\right), j=\overline{0, N}$ avec $B\left(y_{0}, r\right) \subset U \subset X_{\delta}$.

Pour tout $k=\overline{1, N}$ on peut construire une suite $j_{0}, \ldots, j_{l}$ telle que $j_{0}=0, j_{l}=k$ et

$$
B\left(x_{j_{l+1}}, r\right) \subset B\left(x_{j_{l}}, 3 r\right) \text { pour } l=\overline{0, k-1} \text { avec } y_{j_{l}}=\left(s_{j_{l}}, x_{j_{l}}\right) .
$$

Soit $\psi(s, x)=\left|s-s_{j}\right|^{2}+\left|x-x_{j}\right|^{2}$, en vertu de la Proposition 2.2.4, on a pour $\gamma>\gamma_{1}, f \in C_{\bar{V}}^{\infty}\left(\mathbb{R}_{+}^{n+1}\right)$

$$
C\left\|\mathrm{e}^{\gamma \mathrm{e}^{-\beta \psi(s, x)}} f\right\|_{H^{1}(V)} \leq\left\|\mathrm{e}^{\gamma \mathrm{e}^{-\beta \psi(s, x)}} Q f\right\|_{L^{2}(V)} .
$$

On pose $r_{1}=\frac{r}{4}, r_{1}^{\prime}=\frac{r}{2}, r_{2}=\frac{3 r}{4}<r, r_{2}^{\prime}=\frac{7 r}{2}, r_{3}=4 r, r_{3}^{\prime}=5 r$.

Introduisons la fonction de troncature $\tilde{\chi}$ de $\theta$ définie par

$$
\tilde{\chi}(\theta)=\left\{\begin{array}{l}
1 ; \quad \frac{r^{2}}{4} \leq \theta \leq 16 r^{2} \\
0 ; \quad \theta \leq \frac{r^{2}}{16} \text { ou } \theta \geq 25 r^{2},
\end{array}\right.
$$


et considérons $\chi \in C_{\bar{V}}^{\infty}\left(\mathbb{R}^{n+1}\right)$ définie par $\chi(y)=\widetilde{\chi}(\psi(y))$, on prend $f=\chi v$ et de (2.3.10) on obtient en écrivant

$$
\widetilde{Q} f=\chi \widetilde{Q} v+[\widetilde{Q}, \chi] v
$$

$\left([\widetilde{Q}, \chi]\right.$ est un opérateur différentiel d'ordre 1 supporté sur $\left.V_{1} \cup V_{3}\right)$

$$
C \mathrm{e}^{\gamma \mathrm{e}^{-\beta r_{2}^{\prime}}}\|v\|_{H^{1}\left(V_{2}\right)} \leq \mathrm{e}^{\gamma \mathrm{e}^{-\beta r_{1}}}\left[\|Q v\|_{L^{2}(V)}+\|v\|_{H^{1}\left(V_{1}\right)}\right]+\mathrm{e}^{\gamma \mathrm{e}^{-\beta r_{3}}}\|v\|_{H^{1}(V)} .
$$

On pose $C_{1}=\mathrm{e}^{-\beta r_{2}^{\prime}}-\mathrm{e}^{-\beta r_{3}}>0$ et $C_{2}=\mathrm{e}^{-\beta r_{1}}-\mathrm{e}^{-\beta r_{2}^{\prime}}>0$, on obtient

$$
\|v\|_{H^{1}\left(V_{2}\right)} \leq C\left[\mathrm{e}^{\gamma C_{2}}\left(\|Q v\|_{L^{2}(V)}+\|v\|_{H^{1}\left(V_{1}\right)}\right)+\mathrm{e}^{\gamma C_{1}}\|v\|_{H^{1}(V)}\right] .
$$

On optimise en $\gamma$ (comme dans [28]), on a alors

$$
\|v\|_{H^{1}\left(V_{2}\right)} \leq C\left(\|Q v\|_{L^{2}(V)}+\|v\|_{H^{1}\left(V_{1}\right)}\right)^{\mu}\left(\|v\|_{H^{1}(V)}\right)^{1-\mu}
$$

avec $\mu \leq \mu_{0}=\frac{C_{1}}{C_{1}+C_{2}}$.

Par ailleurs on a $V_{1} \subset B\left(y_{j}, r\right)$ et $B\left(y_{j}, 3 r\right) \subset V_{2} \cup B\left(y_{j}, r\right)$, alors (2.3.11) devient

$$
\|v\|_{H^{1}\left(B\left(y_{j_{l}}, 3 r\right)\right)} \leq C\left(\|Q v\|_{L^{2}(V)}+\|v\|_{H^{1}\left(B\left(y_{j_{l}}, r\right)\right)}\right)^{\mu}\left(\|v\|_{H^{1}(V)}\right)^{1-\mu} .
$$

En vertu de (2.3.9), (2.3.12) on a

$$
\|v\|_{H^{1}\left(B\left(y_{j_{l+1}}, r\right)\right)} \leq C\left(\|Q v\|_{L^{2}(X)}+\|v\|_{H^{1}\left(B\left(y_{j_{l}}, r\right)\right)}\right)^{\mu}\left(\|v\|_{H^{1}(X)}\right)^{1-\mu} .
$$

On applique le lemme de récurrence à (2.3.13) avec $\alpha_{l}=\|v\|_{H^{1}\left(B\left(x_{j_{l}}, r\right)\right)}, E=\|Q v\|_{L^{2}(X)}$ et $F=C^{\frac{1}{1-\mu}}\|v\|_{H^{1}(X)}$, on a alors

$$
\|v\|_{H^{1}\left(B\left(y_{k}, r\right)\right)} \leq C\left(\|Q v\|_{L^{2}(X)}+\|v\|_{H^{1}\left(B\left(y_{0}, r\right)\right)}\right)^{\mu^{\prime}}\left(\|v\|_{H^{1}(X}\right)^{1-\mu^{\prime}} .
$$

En recollant les inégalité obtenues, on obtient pour un certain $\mu \in] 0,1[$

$$
\|v\|_{H^{1}\left(X_{\delta}\right)} \leq C\left[\|Q v\|_{L^{2}(X)}+\|v\|_{H^{1}(U)}\right]^{\mu}\left(\|v\|_{H^{1}(X)}\right)^{1-\mu} .
$$

D'autre part, soit $y \in Z=\left[-\frac{s_{0}}{2}, \frac{s_{0}}{2}\right] \times \partial \Omega$, il existe alors un voisinage de $y$ noté $U$ et un difféomorphisme noté $G$ défini de $U \cap X$ vers $B\left(0, r_{y}\right) \cap\left\{x_{n} \geq 0\right\}$ tels que $G(y)=0$; soit $\psi(s, x)=s^{2}+\left|x^{\prime}\right|^{2}+\left|x_{n}-4 r\right|^{2}$ avec $r \ll r_{y}$.

En vertu de la Proposition 2.2.3 (en divisant l'inégalité de cette proposition par $\gamma$ ), on a pour $\gamma>\gamma_{1}$, $f \in C_{\bar{V}}^{\infty}\left(\mathbb{R}_{+}^{n+1}\right)$

$$
C\left\|\mathrm{e}^{\gamma \mathrm{e}^{-\beta \psi(s, x)}} f\right\|_{H^{1}(V)} \leq\left\|\mathrm{e}^{\gamma \mathrm{e}^{-\beta \psi(s, x)}} \widetilde{Q} f\right\|_{L^{2}(V)}+\left\|\mathrm{e}^{\gamma \mathrm{e}^{-\beta \psi(s, x)}} \partial_{n} f\right\|_{L^{2}\left(\partial V \cap\left\{x_{n}=0\right\}\right)} .
$$

On pose $r_{1}=\frac{r}{2}, r_{1}^{\prime}=r, r_{2}=2 r, r_{2}^{\prime}=6 r, r_{3}=7 r, r_{3}^{\prime}=8 r$, on vérifie facilement que $B(0, r) \subset V_{2}$.

Introduisons la fonction de troncature $\tilde{\chi}$ de $\theta$ définie par

$$
\widetilde{\chi}(\theta)= \begin{cases}1 ; & r^{2} \leq \theta \leq 49 r^{2} \\ 0 ; & \theta \leq \frac{r^{2}}{4} \text { ou } \theta \geq 64 r^{2}\end{cases}
$$


et considérons $\chi \in C \frac{\infty}{V}\left(\mathbb{R}^{n+1}\right)$ définie par $\chi(s, x)=\tilde{\chi}(\psi(s, x))$, on prend $f=\chi v$ et de (2.3.15) on obtient en écrivant

$$
\widetilde{Q} f=\chi \widetilde{Q} v+[\widetilde{Q}, \chi] v
$$

$\left([\widetilde{Q}, \chi]\right.$ est un opérateur différentiel d'ordre 1 supporté sur $\left.V_{1} \cup V_{3}\right)$ et

$$
\begin{aligned}
& \partial_{n} f=\chi \partial_{n} v+\chi_{x_{n}}^{\prime} v, \\
& C \mathrm{e}^{\gamma \mathrm{e}^{-\beta r_{2}^{\prime}}}\|v\|_{H^{1}\left(B^{+}(0, r)\right)} \leq \mathrm{e}^{\gamma \mathrm{e}^{-\beta r_{1}}}\left[\|\widetilde{Q} v\|_{L^{2}\left(V^{+}\right)}+\|v\|_{H^{1}\left(V_{1}\right)}\right. \\
& \left.+\left\|\partial_{n} v\right\|_{L^{2}\left(V \cap\left\{x_{n}=0\right\}\right)}\right]+\mathrm{e}^{\gamma \mathrm{e}^{-\beta r_{3}}\|v\|_{H^{1}\left(V^{+}\right)}} .
\end{aligned}
$$

En posant $C_{1}=\mathrm{e}^{-\beta r_{2}^{\prime}}-\mathrm{e}^{-\beta r_{3}}>0$ et $C_{2}=\mathrm{e}^{-\beta r_{1}}-\mathrm{e}^{-\beta r_{2}^{\prime}}>0$, on a

$$
\begin{aligned}
\|v\|_{L^{2}\left(B^{+}(0, r)\right)} \leq & C\left(\mathrm { e } ^ { \gamma C _ { 2 } } \left[\|\widetilde{Q} v\|_{L^{2}\left(V^{+}\right)}+\|v\|_{H^{1}\left(V_{1}\right)}\right.\right. \\
& \left.\left.+\left\|\partial_{n} v\right\|_{L^{2}\left(V \cap\left\{x_{n}=0\right\}\right)}\right]+\mathrm{e}^{-\gamma C_{1}}\|v\|_{H^{1}\left(V^{+}\right)}\right) .
\end{aligned}
$$

On optimise en $\gamma$ (comme dans [28]), on a alors, en posant $\mu_{0}=\frac{C_{1}}{C_{1}+C_{2}}$, pour $\mu \leq \mu_{0}$

$$
\|v\|_{H^{1}\left(B^{+}(0, r)\right)} \leq C\left(\|\widetilde{Q} v\|_{L^{2}\left(V^{+}\right)}+\|v\|_{H^{1}\left(V_{1}\right)}+\mid \partial_{n} v \|_{L^{2}\left(V \cap\left\{x_{n}=0\right\}\right)}\right)^{\mu} \cdot\left(\|v\|_{H^{1}\left(V^{+}\right)}\right)^{1-\mu} .
$$

Puisque $G^{-1}\left(V_{1}\right) \subset X_{\delta_{y}}$ et $\left(B\left(y, r_{y}^{\prime}\right) \cap X\right) \subset G^{-1}(B(0, r)) \cap\left\{x_{n} \geq 0\right\}$ avec $r_{y}^{\prime}$ assez petit, $\widetilde{Q}$ (resp. $\left.\partial_{n}\right)$ se transforme par $G$ en $Q$ (resp. $B$ ) et en utilisant le fait que $G$ transforme les normes en normes équivalentes, on a

$$
\|v\|_{H^{1}\left(B\left(y, r_{y}^{\prime}\right)\right)} \leq C\left(\|Q v\|_{L^{2}(X)}+\|v\|_{H^{1}\left(X_{\delta_{y}}\right)}+\|B v\|_{L^{2}(\partial X)}\right)^{\mu_{y}} \cdot\left(\|v\|_{H^{1}(X)}\right)^{1-\mu_{y}} .
$$

On recouvre $Z$ par un nombre fini de boules de rayon $r_{j}^{\prime}=r_{y_{j}}^{\prime}$ et de centre $y_{j}=\left(s_{j}, x_{j}\right), j=\overline{0, M}$, on a d'après (2.3.16)

$$
\|v\|_{H^{1}\left(B\left(y_{j}, r_{j}^{\prime}\right)\right)} \leq C\left(\|Q v\|_{L^{2}(X)}+\|v\|_{H^{1}\left(X_{\delta_{y_{j}}}\right)}+\|B v\|_{L^{2}(\partial X)}\right)^{\mu_{y_{j}}} \cdot\left(\|v\|_{H^{1}(X)}\right)^{1-\mu_{y_{j}}} .
$$

On pose $\delta_{1}=\inf _{j \in\{0, \ldots, M\}}\left\{\delta_{y_{j}}\right\}$ et $\mu_{1}=\inf _{j \in\{0, \ldots, M\}}\left\{\mu_{y_{j}}\right\}$. (2.3.17) implique

$$
\|v\|_{H^{1}\left(B\left(y_{j}, r_{j}^{\prime}\right)\right)} \leq C\left(\|Q v\|_{L^{2}(X)}+\|v\|_{H^{1}\left(X_{\delta_{1}}\right)}+\|B v\|_{L^{2}(\partial X)}\right)^{\mu_{1}} \cdot\left(\|v\|_{H^{1}(X)}\right)^{1-\mu_{1}} .
$$

En recollant les inégalités de (2.3.18) et en utilisant

$$
\|v\|_{H^{1}\left(X_{\frac{\delta}{2}}\right)}=\|v\|_{H^{1}\left(X_{\frac{\delta}{2}}\right)}^{\mu_{1}}\|v\|_{H^{1}\left(X_{\frac{\delta}{2}}\right)}^{1-\mu_{1}}
$$

c'est-à-dire

$$
\|v\|_{H^{1}\left(X_{\frac{\delta}{2}}\right)} \leq C\left(\|Q v\|_{L^{2}(X)}+\|v\|_{H^{1}\left(X_{\frac{\delta}{2}}\right)}+\|B v\|_{L^{2}(\partial X)}\right)^{\mu_{1}}\left(\|v\|_{H^{1}(X)}\right)^{1-\mu_{1}}
$$

et que $Y=\left[-\frac{s_{0}}{2}, \frac{s_{0}}{2}\right] \times \Omega \subset X_{\frac{\delta}{2}} \cup \cup_{j=1}^{M} B\left(y_{j}, r_{j}^{\prime}\right) \subset X$, on obtient

$$
\|v\|_{H^{1}(Y)} \leq C\left(\|Q v\|_{L^{2}(X)}+\|v\|_{H^{1}\left(X_{\delta_{2}}\right)}+\|B v\|_{L^{2}(\partial X)}\right)^{\mu} \cdot\left(\|v\|_{H^{1}(X)}\right)^{1-\mu}
$$

avec $\delta_{2}=\inf \left\{\delta_{1}, \frac{\delta}{2}\right\}$ et $\mu \leq \mu_{1}$; en combinant l'inégalité (2.3.14) et (2.3.19), on obtient l'inégalité (2.1.4). 


\section{RAPPEL SUR LES FONCTIONS F.B.I.}

\subsection{Fonctions F.B.I.}

Soient $s_{0}>0$ et $r>0$, on pose

$$
v_{a, \lambda, T}(s, x)=\sqrt{\frac{\lambda}{2 \pi}} \int \mathrm{e}^{-\frac{\lambda}{2}(i s+a-t)^{2}} \chi_{T}(t) u(t, x) \mathrm{d} t
$$

avec $\lambda \gg 1, a>0$, et

$$
\chi_{T}(t)= \begin{cases}1 ; & |t| \leq(1-\epsilon) T \\ 0 ; & |t| \geq\left(1-\frac{\epsilon}{2}\right) T .\end{cases}
$$

- Pour simplifier les notations, on pose $v_{a, \lambda, T}=v_{\lambda}$.

On note $\Omega_{s_{0}}=\left(-s_{0}, s_{0}\right) \times \Omega, \Gamma_{s_{0}}=\left(-s_{0}, s_{0}\right) \times \Gamma, \Omega_{T}=(0, T) \times \Omega$ et $\Gamma_{T}=(0, T) \times \Gamma$.

Propriétés 3.1.1. $v_{a, \lambda, T}$ possède les propriétés suivantes :

$$
\begin{gathered}
\lim _{\lambda \rightarrow+\infty} v_{a, \lambda, T}(0, x)=u(a, x) \quad \text { si } \quad|a|<T \\
\left\|v_{\lambda}\right\|_{H^{1}\left(\Omega_{s_{0}}\right)}^{2} \leq C \mathrm{e}^{s_{0}^{2} \lambda}\|u\|_{H^{1}\left(\Omega_{T}\right)}^{2} \\
\left\|Q v_{\lambda}\right\|_{L^{2}\left(\Omega_{s_{0}}\right)}^{2} \leq C \mathrm{e}^{s_{0}^{2} \lambda-\lambda[T(1-\epsilon)-|a|]^{2}}\|u\|_{H^{1}\left(\Omega_{T}\right)}^{2} \\
\left\|\left.v_{\lambda}\right|_{\Gamma_{s_{0}}}\right\|_{L^{2}\left(\Gamma_{s_{0}}\right)}^{2} \leq C \mathrm{e}^{s_{0}^{2} \lambda}\left\|\left.u\right|_{\Gamma_{T}}\right\|_{L^{2}\left(\Gamma_{T}\right)}^{2} \\
\left\|B v_{\lambda}\right\|_{L^{2}\left(\Gamma_{s_{0}}\right)}^{2} \leq C \mathrm{e}^{s_{0}^{2} \lambda}\|B u\|_{L^{2}\left(\Gamma_{T}\right)}^{2} .
\end{gathered}
$$

Remarque 3.1.2. La preuve de ces propriétés est immédiate, par l'inégalité de Cauchy-Schwartz.

Preuve du Théorème 1.0.1. On va utiliser l'inégalité (2.1.2) à $v_{\lambda}$ et on injecte les inégalités (3.1.1)-(3.1.4), on obtient

$$
\left\|v_{\lambda}\right\|_{H^{1}(Y)}^{2} \leq C \mathrm{e}^{s_{0}^{2} \lambda}\|u\|_{H^{1}\left(\Omega_{T}\right)}^{2(1-\mu)}\left(\mathrm{e}^{-\lambda[T(1-\epsilon)-|a|]^{2}}\|u\|_{H^{1}\left(\Omega_{T}\right)}^{2}+\left\|\left.u\right|_{\Gamma_{T}}\right\|_{L^{2}\left(\Gamma_{T}\right)}^{2}\right)^{\mu} .
$$

Ensuite en appliquant la formule $a^{1-\mu} b^{\mu} \leq(1-\mu) a+\mu b$, pour tout $a, b$ dans $\mathbb{R}_{+}^{*}$ et $\left.\mu \in\right] 0,1[$ à $(3.1 .5)$, on trouve

$$
\left\|v_{\lambda}\right\|_{H^{1}(Y)}^{2} \leq C\left[\mathrm{e}^{-\lambda \alpha}\|u\|_{H^{1}\left(\Omega_{T}\right)}^{2}+\mathrm{e}^{\lambda \beta}\left\|\left.u\right|_{\Gamma_{T}}\right\|_{L^{2}\left(\Gamma_{T}\right)}^{2}\right]
$$

où $\alpha=-s_{0}^{2}+(T(1-\epsilon)-|a|)^{2}$ et $\beta=s_{0}^{2}$.

\subsection{Estimation de $u$}

On sait déjà, d'après les Propriétés 3.1.1, qu'on a la convergence simple

$$
\lim _{\lambda \longrightarrow+\infty} v_{\lambda}(0, x)=u(a, x) .
$$

On va préciser cette convergence.

Dans les lemmes qui vont suivre, on va faire abstraction de la variable $x$.

Soit $u(t)$ à support compact

$$
u_{\lambda}(t)=\sqrt{\frac{\lambda}{2 \pi}} \int_{\mathbb{R}} \mathrm{e}^{-\frac{\lambda}{2}(a-t)^{2}} u(t) \mathrm{d} t,
$$

on a alors : 
Lemme 3.2.1. Soit $u \in H^{s}(\mathbb{R}) \cap \mathcal{E}^{\prime}(\mathbb{R})$ (où $\mathcal{E}^{\prime}(\mathbb{R})$ est l'espace des distributions à support compact sur $\left.\mathbb{R}\right)$, alors :

$$
\left\|u-u_{\lambda}\right\|_{L^{2}(\mathbb{R})} \leq \frac{C}{\lambda^{\frac{s}{2}}}\|u\|_{H^{s}(\mathbb{R})} .
$$

Lemme 3.2.2. Pour tout $\epsilon>0$, il existe $C_{\epsilon}>0$ telle que:

$$
\left|u_{\lambda}(a)\right| \leq C_{\epsilon}\left(\int_{\substack{|s| \leq \epsilon \\\left|a-t_{0}\right| \leq \epsilon}}\left|u_{\lambda}\left(t_{0}+i s\right)\right|^{2} \mathrm{~d} t_{0} \mathrm{~d} s\right)^{\frac{1}{2}} .
$$

Lemme 3.2.3. Soit $\vec{u} \in W_{0}$, on a alors :

$$
\|\vec{u}\|_{W_{-1}} \leq\|u\|_{L^{2}\left(\Omega_{T}\right)}^{2}+\frac{C}{\lambda}\left(\|u\|_{H^{1}\left(\Omega_{T}\right)}^{2}+\left\|\partial_{a} u_{\lambda}\right\|_{L^{2}\left(\Omega_{T}\right)}^{2}\right) .
$$

Preuve. La preuve du Lemme 3.2.1 se fait en écrivant $u_{\lambda}$ sous forme de convolution de deux fonctions et la preuve du Lemme 3.2.2 se fait en utilisant la formule de Cauchy. Détaillons la preuve du Lemme 3.2.3 en utilisant [21], p. 311 :

En multipliant scalairement l'équation $u^{\prime \prime}(t)-A u(t)=0$ par $A^{-1} u^{\prime}(t)$, puis en intégrant entre 0 et $t$, on obtient

$$
\|u(0)\|_{L^{2}(\Omega)}^{2}+\left\langle A^{-1} u^{\prime}(0), u^{\prime}(0)\right\rangle=\|u(t)\|_{L^{2}\left(\Omega_{t}\right)}^{2}+\left\langle A^{-1} u^{\prime}(t), u^{\prime}(t)\right\rangle .
$$

Puis en utilisant le fait que

$$
\left\langle A^{-1} v, v\right\rangle \geq c\|v\|_{\left(H^{1}(\Omega)\right)^{\prime}}^{2}, \forall v \in\left(H^{1}(\Omega)\right)^{\prime},
$$

(3.2.1) devient

$$
\|\vec{u}\|_{W_{-1}}^{2}=\|u(0)\|_{L^{2}(\Omega)}^{2}+\left\|u^{\prime}(0)\right\|_{\left(H^{1}(\Omega)\right)^{\prime}}^{2} \leq\|u(t)\|_{L^{2}\left(\Omega_{t}\right)}^{2}+\left\langle A^{-1} u^{\prime}(t), u^{\prime}(t)\right\rangle
$$

on a

$$
\begin{aligned}
\left|\left\langle A^{-1} u^{\prime}(t), u^{\prime}(t)\right\rangle\right| \leq & \left|\left\langle A^{-1} u^{\prime}(t), u^{\prime}(t)\right\rangle-\left\langle A^{-1} u_{\lambda}^{\prime}(a), u_{\lambda}^{\prime}(a)\right\rangle\right| \\
& +\left|\left\langle A^{-1} u_{\lambda}^{\prime}(a), u_{\lambda}^{\prime}(a)\right\rangle\right| .
\end{aligned}
$$

D'une part on a

$$
\left|\left\langle A^{-1} u_{\lambda}^{\prime}(a), u_{\lambda}^{\prime}(a)\right\rangle\right| \leq C\left\|u_{\lambda}^{\prime}\right\|_{L^{2}\left(\Omega_{T}\right)}^{2} ; C>0
$$

car $A^{-1} \in \mathcal{L}\left(\left(H^{1}(\Omega)\right)^{\prime}, H^{1}(\Omega)\right)$; d'autre part on pose $A^{-1} u^{\prime}=v$ et $A^{-1} u_{\lambda}^{\prime}=v_{\lambda}$, on a alors

$$
\left|\left\langle A^{-1} u^{\prime}(t), u^{\prime}(t)\right\rangle-\left\langle A^{-1} u_{\lambda}^{\prime}(a), u_{\lambda}^{\prime}(a)\right\rangle\right| \leq\left|\left\langle v, u^{\prime}\right\rangle-\left\langle v, u_{\lambda}^{\prime}\right\rangle\right|+\left|\left\langle v, u_{\lambda}^{\prime}\right\rangle-\left\langle v_{\lambda}, u_{\lambda}^{\prime}\right\rangle\right| .
$$

En utilisant l'inégalité de Cauchy-Schwartz et le Lemme 3.2.1, on a

$$
\left|\left\langle v-v_{\lambda}, u_{\lambda}^{\prime}\right\rangle\right| \leq \frac{C}{\lambda^{\frac{1}{2}}}\|u\|_{H^{1}\left(\Omega_{T}\right)}\left\|u_{\lambda}^{\prime}\right\|_{L^{2}\left(\Omega_{T}\right)}
$$

et

$$
\left|\left\langle v, u^{\prime}-u_{\lambda}^{\prime}\right\rangle\right| \leq C\|v\|_{H^{2}(\mathbb{R})}\|\| u^{\prime}-u_{\lambda}^{\prime} \|_{H^{-2}(\mathbb{R})} .
$$

En écrivant $\widehat{u}_{\lambda}$ sous forme de convolution de deux fonctions et en remarquant que $\sup _{\xi \in \mathbb{R}}\left|\frac{1-\mathrm{e}^{-\frac{1}{2 \lambda} \cdot \xi^{2}}}{1+|\xi|^{2}}\right| \leq \frac{c}{\lambda} ; c>0$, on a alors

$$
\left|\left\langle v, u^{\prime}-u_{\lambda}^{\prime}\right\rangle\right| \leq \frac{C}{\lambda}\|u\|_{H^{1}\left(\Omega_{T}\right)}^{2}
$$


En vertu de (3.2.3)-(3.2.6), on a le Lemme 3.2.3.

- Revenons maintenant à notre problème :

Soit $T_{0}>0$ et soit $T_{1}>0$ tel que $T_{1}=(1-\epsilon) T_{0}$ avec $T_{1}>T>T_{0}$, on a

$$
v_{\lambda}(0, x)=\sqrt{\frac{\lambda}{2 \pi}} \int_{\mathbb{R}} \mathrm{e}^{-\frac{\lambda}{2}(a-t)^{2}} \chi_{T}(t) u(t, x) \mathrm{d} t=u_{\lambda}(a, x)
$$

et

$$
\begin{aligned}
\|u\|_{L^{2}\left(\Omega_{T_{0}}\right)}^{2} & \leq \int_{\Omega} \int_{\mathbb{R}}\left|u(t, x) \chi_{T}(t)\right|^{2} \mathrm{~d} t \mathrm{~d} x \\
& \leq\left\|u \chi_{T}(a)-v_{\lambda}(0)\right\|_{L^{2}\left(\mathbb{R}_{a} \times \Omega\right)}^{2}+\left\|v_{\lambda}(0)\right\|_{L^{2}\left(\mathbb{R}_{a} \times \Omega\right)}^{2},
\end{aligned}
$$

alors

$$
\|u\|_{L^{2}\left(\Omega_{T_{0}}\right)} \leq\left\|u \chi_{T}(a)-v_{\lambda}(0)\right\|_{L^{2}\left(\mathbb{R}_{a} \times \Omega\right)}+C\left\|\chi_{T_{1}}(a) v_{\lambda}(0)\right\|_{L^{2}\left(\mathbb{R}_{a} \times \Omega\right)}^{2} .
$$

Le premier terme de l'inégalité (3.2.7) est majoré grâce au Lemme 3.2.1 par :

$$
\frac{C}{\sqrt{\lambda}}\|u\|_{H^{1}\left(\Omega_{T}\right)}
$$

Le deuxième terme de l'inégalité ( 3.2.7) est majoré grâce au Lemme 3.2.2 et à l'inégalité ( 3.1.6) par :

$$
\left.C\left[\mathrm{e}^{-\lambda \alpha}\|u\|_{H^{1}\left(\Omega_{T}\right)}^{2}\right)+\mathrm{e}^{\lambda \alpha}\left\|\left.u\right|_{\Gamma_{T}}\right\|_{L^{2}\left(\Gamma_{T}\right)}^{2}\right]^{\frac{1}{2}}
$$

d'où (3.2.7) devient

$$
\|u\|_{L^{2}\left(\Omega_{T_{0}}\right)} \leq \frac{C}{\sqrt{\lambda}}\|u\|_{H^{1}\left(\Omega_{T}\right)}+C \mathrm{e}^{\alpha \frac{\lambda}{2}}\left\|\left.u\right|_{\Gamma_{T}}\right\|_{L^{2}\left(\Gamma_{T}\right)}+\mathrm{e}^{-\frac{\lambda}{2} \alpha}\|u\|_{H^{1}\left(\Omega_{T}\right)} .
$$

D'autre part on a

$$
\partial_{a} u_{\lambda}(a, x)=i \partial_{s} v_{\lambda}(0, x)
$$

En utilisant le Lemme 3.2.2 et l'inégalité 3.1.6, on a alors

$$
\left.\left\|\partial_{a} u_{\lambda}\right\|_{L^{2}\left(\Omega_{T}\right)} \leq C\left[\mathrm{e}^{-\lambda \alpha}\|u\|_{H^{1}\left(\Omega_{T}\right)}^{2}\right]+\mathrm{e}^{\lambda \alpha}\left\|\left.u\right|_{\Gamma_{T}}\right\|_{L^{2}\left(\Gamma_{T}\right)}^{2}\right]^{\frac{1}{2}}
$$

en posant

$$
\lambda=\frac{2}{\mu} \log \left(\frac{\|u\|_{H^{1}\left(\Omega_{T}\right)}}{\left\|u 1_{\Gamma_{T}}\right\|_{L^{2}\left(\Gamma_{T}\right)}}+2\right)
$$

on trouve

$$
\|u\|_{L^{2}\left(\Omega_{T_{0}}\right)}+\left\|\partial_{a} u_{\lambda}\right\|_{L^{2}\left(\Omega_{T}\right)} \leq C\|u\|_{H^{1}\left(\Omega_{T}\right)}\left[\log \left(\frac{\|u\|_{H^{1}\left(\Omega_{T}\right)}}{\left\|u 1_{\Gamma_{T}}\right\|_{L^{2}\left(\Gamma_{T}\right)}}+2\right)\right]^{-\frac{1}{2}} .
$$

Ceci termine la preuve du Théorème 1.0.1 grâce au Lemme 3.2.3. 
Remerciements. Je voudrais exprimer ma profonde gratitude à mon directeur de recherche, Professeur L. Robbiano, pour ses précieuses suggestions qui ont significativement contribué à donner à cet article son contenu, et pour sa lecture critique du manuscrit et ses conseils de rédaction qui ont été très utiles pour le rendre aussi clair et accessible que possible.

\section{RÉFÉRENCES}

[1] S. Alinhac, Non unicité du problème de Cauchy. Ann. Math. 117 (1983) 77-108.

[2] S. Alinhac and M.S. Baouendi, A non uniqueness result for operators of principal type. Math. Z. 220 (1995) 561-568.

[3] H. Bahouri, Dépendence non linéaire des données de Cauchy pour des solutions des équations aux dérivées partielles. J. Math. Pures. Appl. 66 (1987) 127-138.

[4] C. Bardos, G. Lebeau and J. Rauch, Sharp sufficient conditions for the observation, control and stabilization of waves from the boundary. SIAM J. Control Optim. 30 (1992) 1024-1065.

[5] N. Burq, Contrôle de l'équation des plaques en présence d'obstacles strictement convexes. Mém. Soc. Math. France (N. S.) 55, Marseilles (1993).

[6] T. Duyckaerts, Optimal decay rates of the energy of an hyperbolic-parabolic system coupled by an interface. European Union Projects "Smart System" (2002).

[7] T. Duyckaerts, Xu Zhang and E. Zuazua, On the optimality of the observability inequality for parabolic and hyperbolic systems with potentials. Ann. Inst. H. Poincaré Anal. Non Linéaire (to appear).

[8] C. Fabre, Résultats de contrôlabilité exacte interne pour l'équation de Schrödinger et leurs limites asymptotiques : Application à certaines équations de plaques vibrantes. Asym. Anal. 5 (1992) 343-379.

[9] E. Fernandez-Cara and E. Zuazua, The cost of approximate controllability for heat equations: The linear case. Adv. Diff. Equa. 5 (2000) 465-514.

[10] A.V. Fursikov and O. Yu Imanivilov, Controllability of evolution equations. Lect. Notes Ser. 34, Seoul National University, Research Institute of Mathematics, Global Analysis Research Center, Seoul (1996).

[11] L. Hörmander, Linear partiel differential operators. Springer-Verlag, Berlin (1963).

[12] L. Hörmander, On the uniqueness of the Cauchy problem under partial analyticity asumptions. Springer-Verlag (1996).

[13] V.-M. Isakov, On the uniquenss of the solution of the Cauchy problem. Sov. Math. Dokl. 22 (1980) 639-642.

[14] F. John, Continous dependence on data for solution of partial differential equations with prescribed bound. Comm. Pure. Appl. Math. 17 (1960) 551-585.

[15] I. Lasiecka and R. Triggiani, Optimal regularity, exact controllability and uniform stabilisation of Schrödinger equation with Dirichlet control. Diff. Integral. Equa. 5 (1992) 521-535.

[16] G. Lebeau, Contrôle de l'équation de Schrödinger. J. Math. Pures. Appl. 71 (1992) 267-291.

[17] G. Lebeau, Contrôle analytique I : estimation a priori. Duk. Math. J. 68 (1992) 1-30.

[18] G. Lebeau et L. Robbiano, Contrôle exacte de l'équation de la chaleur. Comm Partial. Diff. Equa. 20 (1995) $335-356$.

[19] G. Lebeau et L. Robbiano, Stabilisation de l'équation des ondes par le bord. Duk. Math. J. 86 (1997) 465-491.

[20] J.-L. Lions, Contrôlabilité exacte, perturbations et stabilisation des systèmes distribués. Masson Collection, RMA, Paris (1988).

[21] J.-L. Lions et E. Magenes, Problèmes aux limites non homogènes et applications. Vol. 1, Dunod, Paris (1968).

[22] E. Machtyngier, Exact controllability for Schrödinger equation. SIAM J. Control. Optim. 32 (1994) 24-34.

[23] L. Miller, Geometric bounds on the growth rate of null-controllability cost of the heat equation in small time. J. Diff. Eq. 2004 (2004) 202-226.

[24] L. Miller, On the null-controllability of the heat equation in unbounded domains. Bull. Sci. Math. 129 (2005) $175-185$.

[25] K.-D. Phung, Observability and control of Schrödinger equation. SIAM J. Control Optim. 40 (2001) 211-230.

[26] K.-D. Phung, Note on the cost of the approximate controllability for the heat equation with potentiel. J. Math. Anal. Appl. 295 (2004) $527-538$.

[27] J. Rauch, X. Zhang and E. Zuazua, Polynomial decay for a hyperbolic-parabolic coupled system. J. Math. Pures. Appl. 84 (2005) 407-470.

[28] L. Robbiano, Théorème d'unicité adapté au contrôle des solutions des problèmes hyperboliques. Comm Partial. Diff. Equa. 16 (1991) 789-800.

[29] L. Robbiano, Fonction de coût et contrôle des solutions des équations hyperboliques. Asym. Anal. 10 (1995) 95-115.

[30] L. Robbiano and C. Zuily, Uniqueness in the Cauchy problem for operator with partially holomorphic coefficients. Inventiones Mathematice 131 (1998) 493-539.

[31] D. Russell, A unified boundary contrllability theory for hyperbolic and parabolic partial differential equations. Stud. Appl. Math. 52 (1973) 189-212.

[32] D. Tataru, Unique continuation for solution to P.D.E's between Hörmander theorem and Holmgren's theorem. Comm. Part. Diff. Eq. 20 (1995) 855-884.

[33] D. Tataru, Carleman estimates and unique continuation for solutions to boundary-value problems. J. Math. Pures. Appl. 75 (1996) 367-408. 
[34] D. Tataru, Unique continuation for partial differential operators with partially analytic coefficients. J. Math. Pures. Appl. 78 (1999) 505-521.

[35] X. Zhang and E. Zuazua, Polinomial decay and control of a 1-d model for fluid-structure interaction. C. R. Acad. Sci., Paris, Ser. I 336 (2003) 745-750.

[36] X. Zhang and E. Zuazua, Control, observation and polynomial decay for a coupled heat-wave system. C. R. Acad. Sci., Paris, Ser. I 336 (2003) 823-828.

[37] X. Zhang and E. Zuazua, Long-time behavior of a coupled heat-wave system arising in fluid-structure interaction. Arch. Ration. Mech. Anal. 184 (2007) 49-120. 